\title{
The Feasibility of a Fully Miniaturized Magneto-Optical Trap for Portable Ultracold Quantum Technology
}

\author{
J.A. Rushton, M. Aldous and M.D. Himsworth* \\ School of Physics \& Astronomy, University of Southampton, Southampton, SO17 1BJ, UK
}

(Dated: December 2, 2015)

\begin{abstract}
Experiments using laser cooled atoms and ions show real promise for practical applications in quantum-enhanced metrology, timing, navigation, and sensing as well as exotic roles in quantum computing, networking and simulation. The heart of many of these experiments has been translated to microfabricated platforms known as atom chips whose construction readily lend themselves to integration with larger systems and future mass production. To truly make the jump from laboratory demonstrations to practical, rugged devices, the complex surrounding infrastructure (including vacuum systems, optics, and lasers) also needs to be miniaturized and integrated. In this paper we explore the feasibility of applying this approach to the Magneto-Optical Trap; incorporating the vacuum system, atom source and optical geometry into a permanently sealed micro-litre system capable of maintaining $10^{-10}$ mbar for more than 1000 days of operation with passive pumping alone. We demonstrate such an engineering challenge is achievable using recent advances in semiconductor microfabrication techniques and materials.
\end{abstract}

\section{ULTRACOLD QUANTUM TECHNOLOGY}

Since the first demonstrations of atoms and ions at sub-millikelvin temperatures in the mid-1980s, the field of atomic physics has been revolutionized by laser cooling and trapping as it provides researchers with a method to probe some of the purest, most sensitive, and controllable quantum systems available. This field is still highly productive and recently has put significant emphasis on the practical applications of this technology beyond the laboratory $[1,2]$. It was evident very early on that ultracold matter would be an indispensable tool in precise timing applications and a recent demonstration [3] has shown extremely low instabilities at the $10^{-18}$ level. The wavelike nature of atoms as they are cooled to lower temperatures can be used to form atomic interferometers that outperform their optical counterparts in measurements of accelerated reference frames [4-7], which are important for inertial guidance systems, but can also provide sensitive measurements of mass, charge and magnetic fields [8-11]. Greater sensitivity beyond the classical limit is possible via squeezed states [12], interacting particles [13, 14] and entangled states [15-17], which are also fundamental attributes for quantum computing [18, 19], and long distance quantum networking [20]. Ultracold matter has been used in the emerging field of quantum simulation [21] and is an indispensable tool in determining fundamental constants [22], testing general relativity [23] and defining measurement standards [24]. Many researchers and industries believe such tools will be a major part of the 'second quantum revolution' in which the more 'exotic' properties of quantum physics are applied for practical applications [25, 26].

The field of ultracold matter has reached maturity in both experimental methods and theoretical understanding allowing experiments to begin leaving the laboratory

*m.d.himsworth@soton.ac.uk
[27-29]. These systems are bespoke, rarely take up a volume less than a cubic metre, and require a team of experts to operate. The many applications that will benefit most from ultracold quantum technology are likely to compromise sensitivity for far smaller and more rugged devices, which can be mass-produced and do not require the user to understand the internal operation in detail. Commercial pressure will always push toward reduced size, weight and power. One can already see the opportunities made possible with the move to microfabricated atom and ion traps [30-33], but these firmly remain 'chipin-a-lab' components rather than 'lab-in-a-chip' systems.

The miniaturization we envisage is analogous to that demonstrated by the recent development of commercially available [34] chip-scale atomic clocks (CSACs), which have shrunk a traditionally bulky optical spectroscopic system down to one smaller than a grain of rice [35]. Some work has begun on miniaturizing the entire ultracold atom system, most noteably the backpack-sized iSense gravimeter [36], but to achieve the CSAC level of sophistication, size and robustness in ultracold technology will require at least another decade of development.

The trapping and cooling of hot vapour-phase atoms or ions below millikelvin temperatures is the first stage in all ultracold experiments, therefore the miniaturization of this system known as the Magneto-Optical Trap [37] (MOT) would be a significant step forward towards our goal. Several academic and commercial research groups have begun looking at the various ways the MOT can be miniaturized using machined glass chambers [38], conical retro-reflectors [39, 40], and etched multi-section silicon and glass substrates [41]. Most of these demonstrations are small-scale versions of standard MOTs, with only the last device beginning to redesign the system from a microfabricated and integrated approach.

In this study we explore the feasibility of miniaturizing and integrating the ultra-high vacuum system, atom source and MOT optics into a centimetre-scale device. This will be achieved by using recent advances in ma- 
terials and techniques adapted from the semiconductor and MEMS industries used in wafer-level mass production. We will refer to the device as a 'MicroMOT' because the internal volume is sub-millilitre compared to the typically litre-sized standard MOTs. The initial target operational lifetime is set at 1000 days, as this would be at the lower end of a typical commercial service life whilst still presenting a significant challenge. We also aim to maintain an internal vacuum of $10^{-10}$ mbar under normal atmospheric external conditions, and do so with only passive pumping elements and thus no power. Our objective is to focus on this as an engineering challenge from which a mass-producible technology can be developed, thus avoiding bespoke systems which may only be suitable for proof-of-concept purposes.

In Sections II and III we describe a typical MagnetoOptical Trap system, its construction, and how it can be miniaturized including the lasers and optical systems. In Section IV we discuss the source of vapour phase atoms and how to control them. In Section V we explore solutions to provide pumping, prevent permeation, limit leaks, and overcome outgassing. In Section VI we bring the above technologies together to design a prototype Micro-MOT. In Section VII we discuss the assumptions made in the study and highlight areas for further research.

\section{THE MAGNETO OPTICAL TRAP SYSTEM}

Nearly all cold atom experiments begin with a Magneto Optical Trap of which a typical design comprises an Ultra-High Vacuum (UHV, $<10^{-9}$ mbar) chamber with internal volumes of around a litre with numerous optical ports, atom sources, gauges and pumps attached. UHV is obtained by thorough cleaning of the polished glass and metal (typically stainless steel) components. The entire system is assembled and evacuated using roughing and turbomolecular pumps down to around $10^{-7}$ mbar. It is then baked in the vicinity of $200^{\circ} \mathrm{C}$ for several days whilst being evacuated by ion and sublimation pumps and, once cooled, will obtain vacua in the region of $10^{-10} \mathrm{mbar}$. Obtaining vacua much beyond this, in the extreme high vacuum (XHV) regime, can be very difficult and may require getters, cryogenic pumps, deeper cleaning regimes and alternative chamber materials.

Once UHV is obtained, the MOT is formed of several stabilized and finely-tuned laser beams that are retro-reflected along each Cartesian axis intersecting at the zero of a quadrupole magnetic field (see Figure 1). Vapour-phase atoms are released into the chamber, cooled, trapped, and finally manipulated for their intended task. Typically $10^{7}$ atoms are trapped in a dense cloud with diameters usually below $1 \mathrm{~mm}$ and, for many experiments (excluding ultra-sensitive long freefall experiments), the atoms rarely move more than a few millimetres away from this point. The past decade has seen the emergence of atom chips which allows for manipulation

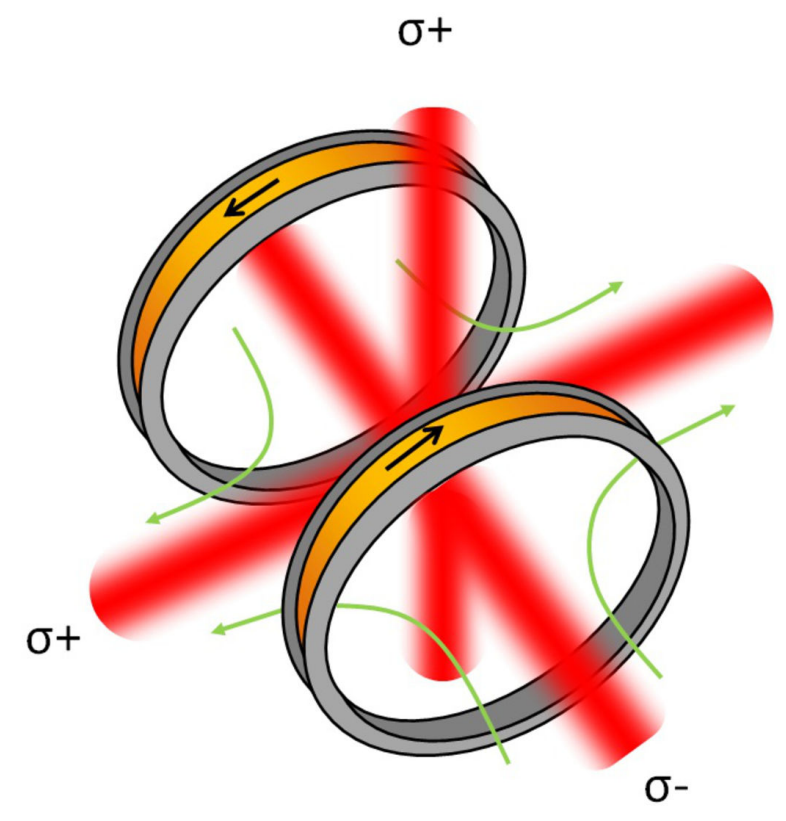

FIG. 1. The standard MOT geometry. The laser polarizations are indicated in text and the magnetic field direction in green arrows.

of atoms microns away from surfaces using high magnetic field gradients, created by microfabricated wires [42].

This raises the question to why such a large vacuum system is required? The answer is that without resorting to bespoke designs the pumps and gauges one can purchase for UHV systems are very large, and regardless, using current approaches the system is still difficult to reduce below the size of a shoebox. Typically these are far too bulky, expensive, and labour intensive to massproduce and so an alternative architecture and manufacturing approach is required, starting with the MOT geometry.

For an integrated device the 'standard' geometry presented above is impractical due to the need for many optical ports, complex alignment, large volumes, numerous fragile optical elements and the difficulty in bringing the atoms close to an atom chip surface. Several alternative geometries have been proposed including the mirror-MOT [43], pyramid-MOT [44, 45], and tetrahedral MOT [46]. The latter two are attractive as they need only a single incident beam, whose phase stability aids in sub-Doppler cooling mechanisms, and both are suitable for microfabrication. Miniaturized pyramid MOTs, however, suffer from low atom capture rates due to the small volume in which the beams overlap [47, 48], significant backscatter making the atoms difficult to detect [49], and the geometry making transfer of the atoms to magnetic surface traps non-trivial. A recently demonstrated planar version of the tetrahedral-MOT using a two dimensional grating as a reflector (which we refer to as the 'G-MOT', see Figure 2) can capture a large number of atoms, has 


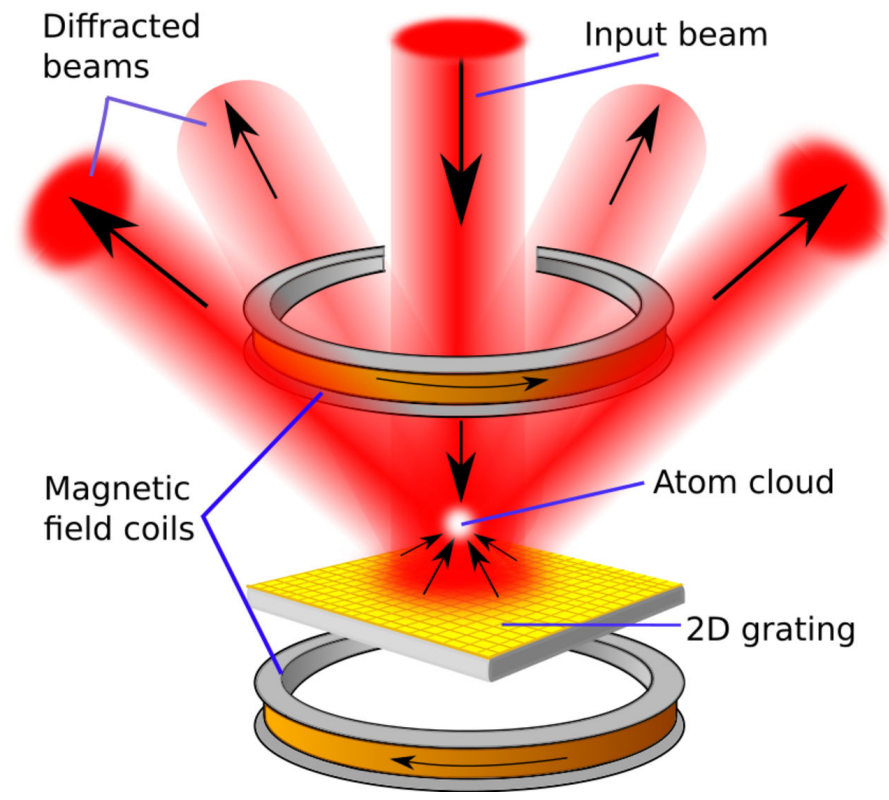

FIG. 2. A grating MOT geometry.

TABLE I. Comparison of the properties of various microfabricated MOT geometries $(\mathrm{M}=$ Mirror, $\mathrm{P}=$ Pyramid, $\mathrm{T}=$ Tetrahedral, $\mathrm{G}=$ Grating, $\mathrm{S}=$ Standard.) compatible with atom chips. Therefore we consider only the small scale pyramid MOTs here, but larger reflectors follow $V^{1.2}$ scaling and are suitable for compact space-borne cold atom systems [54].

\begin{tabular}{|c|c|c|c|c|c|}
\hline MOT & $\mathrm{M}$ & $\mathrm{P}$ & $\mathrm{T}$ & G & $\mathrm{S}$ \\
\hline Number of beams & 4 & 1 & 1 & 1 & 6 \\
\hline Optical ports & 3 & 1 & 1 & 1 & 6 \\
\hline Volume scaling ${ }^{a}$ & $V^{1.2}$ & $V^{2}$ & $V^{1.2}$ & $V^{1.2}$ & $V^{1.2}$ \\
\hline Scatter & low & high & low & medium & none \\
\hline Bandwidth $^{\mathrm{b}}$ & all & all & all & $100 \mathrm{~nm}$ & all \\
\hline Fabricatability & simple $^{\mathrm{c}}$ & difficult & difficult & medium $^{\mathrm{d}}$ & $\mathrm{N} / \mathrm{A}$ \\
\hline
\end{tabular}

${ }^{a}$ Larger exponents lead to lower numbers at smaller volumes.

We include scaling laws for beams diameters greater than $1 \mathrm{~mm}$.

b Limited by surface coatings and windows.

${ }^{\mathrm{c}}$ For the MicroMOT design in Section VI, wide parallel beams are not trivial [55].

d This can be made simple with 'nanoimprint' techniques.

lower backscatter [50], and can be easily integrated with atom chip structures [51]. Some disadvantages include the effect of the grating on the wavefronts and polarizations of the manipulation beams [52], and added difficulty in situations which require several widely-spaced wavelengths (greater than 100nm [53]). Nevertheless, the G-MOT appears to be the most suitable geometry for microfabricated devices. A comparison of the most suitable MOT geometries is given in Table I.

A MOT should trap sufficient atoms to obtain adequate sensitivities for intended application which will ultimately be limited by the quantum projection noise. Typical numbers of atoms range from $10^{4}$ for clock standards [56] to $10^{8}$ atoms for the initial cooling stage into degeneracy. Of course, there exists other parameters which define the performance of cold atom systems, such as coherence time, shot noise, quantum state fidelity, etc. but these factors are somewhat unrelated to the MOT itself where one can only affect the atom number, density, capture rate and temperature. The temperature is typically in the region of tens to hundreds of microkelvin, no matter the MOT geometry if sub-Doppler cooling mechanisms exist, whereas atom number and density are inter-related and are significantly affected by MOT geometry and dimensions. The capture rate depends more on the atom source/loading mechanism and background gas pressure than the MOT geometry and will be discussed in Section IV.

For most cold atom experiments, the practical number of trapped atoms is on the order of $N_{t}=10^{6}$. The GMOT characteristics show that the beam overlap volume, $V_{t}\left(\mathrm{~cm}^{3}\right)$, follows the scaling law of $N_{t}=4 \times 10^{7} V_{t}^{1.2}$, resulting in a minimum practical volume of $0.045 \mathrm{~cm}^{3}$. This is equal to a $0.65 \mathrm{~cm}$ diameter, uniformly illuminated, beam [57] forming a pyramidal volume. To ensure the correct number of atoms and to take into account the effects of non-uniformly shaped beams, a pragmatic beam diameter would be $1 \mathrm{~cm}$. Experiments requiring degenerate gases may require up to $10^{8}$ atoms to ensure a stable phase density for condensation and also improve detection. This would warrant a beam diameter over double that described above.

We assume that the device would incorporate an atom chip structure so that the atoms are trapped and manipulated with magnetic fields close to the surface, therefore the dimensions of the device have little impact on the measurement. Studies have shown that laser cooling close to surfaces begins to show losses as the atom-surface separation decreases below $1 \mathrm{~mm}[49,58]$. Thus, assuming a typical MOT cloud with a diameter less than $1 \mathrm{~mm}$, a lower limit on the vertical dimension would be $3 \mathrm{~mm}$. We aim to explore this issue in another study, but point out here that for a $1 \mathrm{~cm}$ diameter GMOT beam, $94 \%$ of the overlap volume is contained within the first $3 \mathrm{~mm}$ from the grating surface.

In other matterwave experiments which manipulate the atoms during free fall, the interaction time may be limited to several milliseconds in a $3 \mathrm{~mm}$ thick chamber. For example, if we assume our trapped species is rubidium which has been cooled to the Doppler limit of $146 \mu \mathrm{K}$ (r.m.s. speed of $20 \mathrm{~cm} \mathrm{~s}^{-1}$ ), and the atoms have $1 \mathrm{~mm}$ to travel before losses occur, then the maximum interaction time is $5 \mathrm{~ms}$ neglecting gravity. This makes the system applicable to recent work on high repetition rate cold atom intertial sensors [59]. Sub-Doppler cooling can increase the interaction time by a factor of 3 to 4 , approaching the limit imposed by gravitational acceleration to the surface, which may be acceptable in many situations. Alternatively, optical lattices can increase the interaction time of matterwave interferometers without drastically increasing the dimensions of the system [60]. These guided matter waves are a promising technique 
which could aid miniaturization and improve sensitivity.

\section{LASERS AND OPTICS}

In this study we will mainly concentrate on the UHV system as this has had very little development in terms of miniaturization and integration. However, the MOT also requires laser systems, optics, optical modulators, spectrometers, and control electronics which could become the limiting factor to the smallest scale cold atom technology can achieve. A single laser systems can be used to provide optical power to a number of MOTs which might be necessary in multi-axis inertial sensing, or spatial resolved gravity gradiometry; therefore one could accept a larger scale of laser system, but the optics used to couple and expand the beam into the MOTs would need to be significantly reduced in size and made far more robust with the possibility of mass-production. Diode lasers, by their very nature are extremely small and it is the surrounding optics that controls and couples the light to external systems that determines the size of the 'laser system'. Miniaturization can also improve the properties of these lasers by reducing the effects of thermal expansion and vibration of the external components.

Feasible approaches to miniaturize the optics of the laser system - as well as switching, modulating and routing the beams beyond the laser - include optical MEMS, planar optics, precision placed micro-optics, and optical waveguides (including fibers). Exploring each of these fields compehensively is beyond the scope of this report but we shall highlight a few technologies directly suitable for miniaturized MOTs. For this the crucial components to miniaturize and integrate are the laser diodes, wavelength control elements, optical isolators, routing, and beam expansion.

Micro-optical systems for cold atom applications have been pioneered by the Ferdinand-Braun Institut which have integrated diode lasers, Bragg reflectors, isolators, modulators, spectrometers, optical amplifiers and fiber couplers, all on a thermally-stable ceramic substrate no bigger than a credit card [61,62]. They have developed lasers both for the MOT and high power systems [63] for exciting Raman transitions used in the iSense project [36].

The smallest scale devices, and most applicable to mass-production, are planar-waveguide coupled lasers, which are commercially available at the telecoms wavelengths, and show remarkably narrow linewidths and mode-hop free operations along with reasonable powers [64-66]. For cooling rubidium at $780 \mathrm{~nm}$ there is a direct frequency doubling route from $1560 \mathrm{~nm}$ for these lasers and is currently being developed for compact and robust space-based atom interferometers [67]. Waveguide coupled lasers can take advantage of the various chipbased control mechanisms such as phase-modulators [68], coupler/splitters, switches and isolators [69]. There will need to be some development to improve the efficiency of

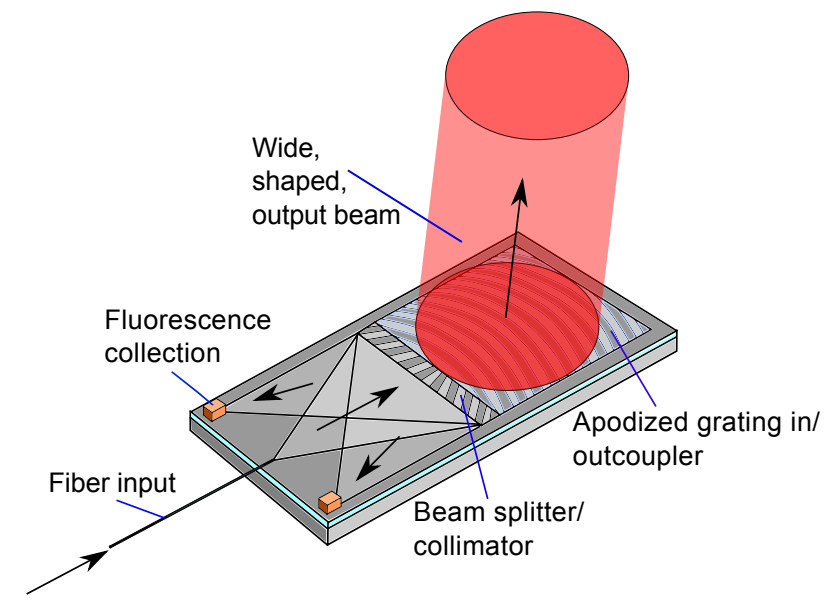

FIG. 3. An integrated confocal microscope adapted from [70]. Such technology can be used to produce large diameter shaped beams from planar waveguides and also provide fluoresence detection.

these systems at short wavelengths where the effect of refractive index inhomogeneities will have a greater impact compared to existing telecoms wavelengths. More development is also required in the areas of chip-based nonreciprocal optical isolators and high-attenuation, highspeed, switches for the demanding constraints imposed by decoherence-free atomic interactions. Integrating several optical elements onto a single substrate will be a sizeable challenge so optical fibre connected elements will likely dominate.

As discussed in the previous section, MOTs require quite large beam diameters which result in large optical systems to expand and collimate beams. We see that a promising route towards miniaturising this is with grating-based waveguide outcouplers. Coupling freespace laser beams into a waveguide is typically achieved with prisms placed on top of the waveguide to enable a reduced wavevector difference between the guided and free-space modes. This can be shrunk into grating couplers which allow one to improve efficiency by altering the dimensions of the grating. Concordantly, this process can be used in reverse to out-couple beams and even focus and shape the beam profile. One directly-applicable example is the work on integrated confocal microscopes [70], as shown in figure 3 , that also provide a means to measure the reflected light intensity, or in our case the fluorescence of the atoms. Due to the diffractive nature of the light coupling the beam profile can be designed with top-hat shapes with flat phase wavefronts - very important properties in efficient atom interferometry using Raman transitions. These grating out-couplers have recently been developed to address individual trapped ions [71]. Planar optics offer alternative methods to expand beams in compact systems using total internal reflection and patterned surfaces within waveguides [72].

For applications which require laser beams access along several axes integrated prisms and reflectors within the 
vacuum chamber are being developed along with integrated windows in silicon substrates [41] and Fresnel lenses [73-75]. Fibre coupling into and out of vacuum would be beneficial for strong coupling to atoms and interconnectable cold-atom systems. Waveguides are, yet again, a viable route for this [76] and possibly recent advances in tapered nano-fibers [77] which can have several alternative applications [78]. The recent development of light-field imaging allows one to perform 3D imaging via computer deconvolution along a single optical axis, thus greatly simplifying the optical system where multiple viewpoints are required [79].

The lasers must be stabilised to a specific closed-cycle resonance of the atoms which are to be trapped. Many techniques are available to produce useful spectroscopic signals and the advances in chip scale atomic clocks [80], hollow waveguides [81], and sub-wavelength fibres [82] and cells [83] provide techniques for coupling beams into atomic vapours. Therefore one can see that miniaturization of the above systems has several clear development route.

\section{ATOM SOURCE AND CONTROL}

The atomic species to be cooled and trapped can be sourced either from a hot vapour, or captured from an atomic beam. The latter is usually produced from a hot Knudsen oven, and requires additional cooling to obtain a suitable capture efficiency in the MOT, usually via a Zeeman slower [84] or chirped cooling [85]. Our chip based system will be far too small for such slowers as they require tens of centimetres for adequate deceleration, although they can be made smaller using bichromatic force techniques [86]. Loading from a background vapour is a common method which results in reasonably fast loading rates, but requires a vapour pressure greater than UHV, resulting in increased collisions and decoherence during subsequent manipulation [87]. Therefore the vapour pressure must be controllable on short times scales, ideally within a second. A common method to achieve this uses a MOT cooled in two dimensions (2D$\mathrm{MOT}$ ) in one chamber separated from a 3D-MOT in another via a narrow conductance channel [88]. The 2DMOT chamber may be kept at a high vapour pressure so that it may load many atoms into a low velocity beam directed into the higher vacuum $3 \mathrm{D}-\mathrm{MOT}$ chamber. This technique has been used in miniaturized BEC systems [41]. Such multichamber systems are likely to be necessary for obtaining BECs which require a higher level of vacuum, however in this study we aim solely to produce a cold non-degenerate cloud of atoms, concentrating on loading a single MOT from a room temperature vapour, and to control it on short timescales. In situations where long coherent times are not required, such as in high data rate interferometers, one can tolerate higher vapour pres- sures and even recapture the cold atom cloud [59].

Each laser cooled species has different chemical properties which bring different challenges. In this study we look at rubidium as it is ubiquitous across the whole scope of cold atom experiments, and poses the challenge of a vapour pressure which is too high at room temperature for efficient trapping. Species with lower vapour pressure, such as strontium, could be easier to use as they do not endanger the vacuum, but the high temperatures needed to obtain a suitable background pressure results in less efficient trap loading, and so may require the additional cooling mechanisms discussed above. Rubidium melts at $39^{\circ} \mathrm{C}$ and at room temperature has a $\left({ }^{87} \mathrm{Rb}\right)$ vapour pressure of $3 \times 10^{-7} \mathrm{mbar}[89]$. This results in significant collisional rates with trapped atoms and also excessive fluorescence, making the detection of the cold atoms very difficult. Moreover, the very small volume of the MicroMOT will quickly result in vapour saturation with no pumping mechanisms. A sufficient vapour pressure to load a rubidium MOT is $\sim 10^{-8} \mathrm{mbar}$, but one must reduce this by an order of magnitude for any decoherence-sensitive measurements. Therefore, one must have a method to carefully regulate the flow of rubidium into the MOT chamber.

The past decade's development of CSACs has provided a range of methods to introduce alkali atoms into microfabricated devices. These sources include pure metal [90, 91], alkali compounds [90], wax pellets [92], alkali azides [93], and alkali-enriched glass [94]. Most are not suitable for UHV or result in poorly controlled, or limited lifetime, sources. Pure rubidium is not suitable unless it is sealed away during fabrication as its high pressure vapour will ruin vacuum at the elevated temperatures required for baking and bonding. Commercial alkali dispensers, such as SAES Getters Alkali Metal Dispensers (AMDs) and Alvatec Alvasources are alkali compounds which are stable up to temperatures of $300-600^{\circ} \mathrm{C}$. AMDs are chromates combined with a $\mathrm{Zr}-\mathrm{Al}$ getter material held in a nichrome dispenser [95]. Heating of the AMDs results in a reduction reaction releasing pure rubidium and some additional gases which are gettered away. Alvasources are alkalis alloyed with 'poor' metals, such as bismuth, which form stable compounds with higher sublimation temperatures than their constituent elements. They also result in far less residual gas than AMDs [96], albeit at a higher cost. Both of these sources can be controlled with Joule heating, but they can also be activated with a focused laser $[97,98]$, removing the need for electrical feedthroughs and reducing the heat transfer to the chip [99].

Rubidium vapour will reach saturation very quickly within micro-litre volumes, especially as the previously mentioned sources may be difficult to control accurately, so a system to pump away the vapour must be incorporated. Glass and metals are effective pumps for alkali atoms: surface studies have found binding energies around $3 \mathrm{eV}$ and extremely high pumping rates [87] of 
$10^{3} 1 \mathrm{~s}^{-1} \mathrm{~cm}^{-2}$. Studies looking at vapour cell coatings [100] have highlighted a significant 'curing time' after filling, during which the vapour pressure stabilizes due to strong chemisorption [101]. After the surface is saturated the adsorption energy drops to $\sim 0.5 \mathrm{eV}$ and is thus only weakly physisorbed. If we assume the MicroMOT produces a 10 second pulse of rubidium every minute, with a peak pressure of $10^{-8} \mathrm{mbar}$, which is pumped away at $11 \mathrm{~s}^{-1}$, one would require a total of $10^{19}$ atoms (about $1 \mathrm{mg}$ ) to last for our 1000 day target. A typical monolayer is around $5 \times 10^{14} \mathrm{~cm}^{-2}$, so one cannot rely on surface pumping alone if it cannot be degassed regularly [102]. We note that the limited surface area can be increased with materials such as aerogel, porous silicon, zeolites, and anodic alumina.

An obvious and effective method to control the vapour is by simply reducing the temperature of the MicroMOT. To get to $10^{-10}$ mbar one must cool rubidium to $-30^{\circ} \mathrm{C}$. This can be accomplished by cooling the entire chip or with an integrated 'cold finger', such as a micro-peltier device [103]. This latter method will avoid rubidium condensation on critical features such as the windows or reflectors, and also avoid water accumulating on external surfaces. The pumping of alkali metals by getter films has been reported to be negligible [104, 105], but little data is available [106], so may not be useful in its regulation. Many atom chips require gold films for reflective surfaces and conductors and it is known in the field that these may degrade over time when exposed to a hot rubidium source. The phase diagram [107] between gold and rubidium shows a stable alloy forms around $500^{\circ} \mathrm{C}$. Therefore one can use a heated gold surface to pump away excess rubidium [108]. Another method could utilize the rubidium/bismuth alloying effect mentioned earlier as a thermally controlled pump, but one must be wary of the low melting point of this metal $\left(271^{\circ} \mathrm{C}\right)$ during fabrication. Both alloying methods work for all alkali metals but, as shown in Table II, these occur at different temperatures. A common getter for caesium in frequency standards is polycrystalline graphite [109]. There exists extensive theoretical work due to the recent interest in graphene electronics [110], but limited data on the adsorption and intercalation of alkalis with graphite (with the exception of potassium [111]), however the heavier alkalis display similar charateristics and therefore this type of getter could be applicable.

A common method to quickly control the vapour pressure whilst remaining at room temperature is Light Induced Atomic Desorption (LIAD) [112]. This technique involves the illumination of metal or glass surfaces with non-resonant ultraviolet light (UV) in order to increase the desorption rate of physisorbed alkali atoms. The exact mechanism by which this occurs is still under debate [113-115]. Once the UV light is extinguished the desorption rate reduces so that atoms can return to the surfaces. This reloading of the atom sources means that the total number of atoms in the device can be reduced through recycling. Studies have shown an order of magnitude
TABLE II. Gold and bismuth alkali alloys with 1:1 compositions for use as alkali pumping mechanisms. Many of the phase diagrams exhibit several phases with additional alloys forming above and below these temperatures and the reader should refer to the original sources. The approximate values are due to indistinct alloying temperatures.

\begin{tabular}{lcc}
\hline $\begin{array}{l}\text { Alkali } \\
\text { metal }(\mathrm{M})\end{array}$ & $\begin{array}{c}\mathrm{Au}-\mathrm{M} \text { alloy } \\
{ }^{\circ} \mathrm{C}\end{array}$ & $\begin{array}{c}\text { Bi-M alloy } \\
{ }^{\circ} \mathrm{C}\end{array}$ \\
\hline $\mathrm{Li}[122,123]$ & $\sim 660$ & $\sim 400$ \\
$\mathrm{Na}[124,125]$ & 372 & 444 \\
$\mathrm{~K}[126,127]$ & 532 & 355 \\
$\mathrm{Rb}[107,128]$ & 498 & 376 \\
$\mathrm{Cs}[129,130]$ & 585 & 390 \\
\hline
\end{tabular}

improvement of MOT loading rates with this technique $[116,117]$, and it has been used to make BECs, which are very sensitive to background gas collisions, in a single chamber [118]. In chip-scale systems the surface area is far too small for effective use of LIAD [119] but, as mentioned earlier, one can introduce high surface area materials $[120,121]$ providing they can be degassed sufficiently prior to encapsulation.

For any pumping mechanism the production of rubidium from the source should be well controlled to ensure consistent loading of the MOT and to prevent permanent vapour saturation. If the source reactively produces hot vapour at unpredictable rates, due to material or heating inhomogeneities, then additional mechanisms are needed to control the flow. Separating two chambers of different pressures is a common challenge in UHV systems, as discussed earlier in 2D/3D MOT loading, and can be achieved by carefully limiting the gas conductance between them with a narrow channel. A channel $1 \mathrm{~mm}$ long with a cross-section of $100 \times 100 \mu \mathrm{m}$, can maintain UHV in the MicroMOT chamber at room temperature [131] whilst the source chamber is at saturation pressure, as long as there is a pumping rate greater than $0.1 \mathrm{ls}^{-1}$ in the laser cooling chamber. Locally heating the source chamber by $100^{\circ} \mathrm{C}$ will sufficiently increase the vapour pressure for loading the MOT. The narrow aperture also leads to a 'beaming effect' which may aid the loading of the trap.

\section{UHV IN A CHIP}

Table III highlights the various challenges in terms of leak, permeation, and outgassing rates that must be tackled to realize sealed passive UHV chips, and compares them to those required by typical UHV systems. Reaching many of these values, especially those for noble gases, may seem unachievable, however we have identified methods to do so by careful choice of materials, fabrication processes and also structural features.

Vacuum encapsulation of microfabricated devices is a large and mature industry and nearly all MEMS devices require some level of hermetic sealing. The range of 
TABLE III. General characteristics of standard UHV MOT systems, and those for the MicroMOT

\begin{tabular}{|c|c|c|}
\hline & Standard & MicroMOT \\
\hline Internal volume (l) & $>1$ & $<10^{-3}$ \\
\hline Lifetime (days) & indefinite & 1000 (target) \\
\hline Pump rate $\left(1 \mathrm{~s}^{-1}\right)$ & $>20$ & $<1$ \\
\hline Leak rate $\left(\right.$ mbar $\left.^{\prime} \mathrm{s}^{-1}\right)$ & $<10^{-11}$ & $\begin{array}{l}<10^{-19}(\mathrm{Ar}) \\
<10^{-14}\left(\mathrm{~N}_{2}\right)\end{array}$ \\
\hline $\begin{array}{l}\text { Outgassing rate } \\
\left(\mathrm{mbar}_{\mathrm{ls}}^{-1} \mathrm{~cm}^{-2}\right)\end{array}$ & $<10^{-11}$ & $\begin{array}{l}<10^{-21}(\mathrm{He}) \\
<10^{-16}\left(\mathrm{H}_{2}\right)\end{array}$ \\
\hline Permeation rate $^{\mathrm{a}}\left(\mathrm{cm}^{2} \mathrm{~s}^{-1}\right)$ & $<10^{-7}$ & $<10^{-17}$ \\
\hline
\end{tabular}

${ }^{\text {a }}$ For helium using Equations 2 and 5.

vacuum levels required ranges from $10^{2}$ mbar in MEMS accelerometers to $10^{-4}$ mbar in microbolometers [132]. Very low vacua are also needed in field emission devices and the lowest recorded encapsulated pressure the authors have found in the literature, $10^{-8}$ mbar [133], was achieved using this technology. Maintaining UHV is also important to photomultiplier tubes and we highlight the work of Erjavec [134] who have performed a similar study to this one. Lower pressures in encapsulated microdevices have probably been achieved, but the means to measure them do not exist as most gauges with capability down to UHV have far greater internal volumes than the devices themselves. We are fortunate that the device we are aiming to produce, by its very nature, is capable of measuring such low pressures. It is commonly known in the atom trapping field that the loading rate, $\gamma(\mathrm{Hz})$, of an atom cloud is linearly related to the background pressure, with an approximate scaling of $2 \times 10^{-8} \gamma$ mbars, and Arpornthip et al [87] performed a systematic study of this gauging technique. It was found to vary little with systematic variations, such as cooling beam power and detuning, and had a sensitivity range from $10^{-7}$ to below $10^{-9}$ mbar, limited by collisions between cold atoms within the trapped cloud. This sensitivity range is slightly above the range of our target pressure but will provide an adequate indication of the internal environment. An improved sensitivity down to $10^{-12}$ mbar may be possible if the background rubidium vapour can be quickly reduced after loading [135] using the techniques discussed in the previous section.

Two methods are commonly used to encapsulate low pressures inside chips [132]: wafer to wafer bonding under vacuum, or sealing of an evacuation tube after bonding (as shown in Figure 4). The latter is simpler to construct as the various chip layers do not need to be manipulated and bonded under UHV. This method has been used in the NIST atom chip system in which the evacuation tube is constantly pumped by a miniature ion pump. However, efficient evacuation to UHV through a small aperture is difficult and the sealing process is non-trivial on a wafer-level scale in terms of complexity, uniformity and time. We shall see that wafer-to-wafer bonding under vacuum, whilst complicated to implement, allows one to a)

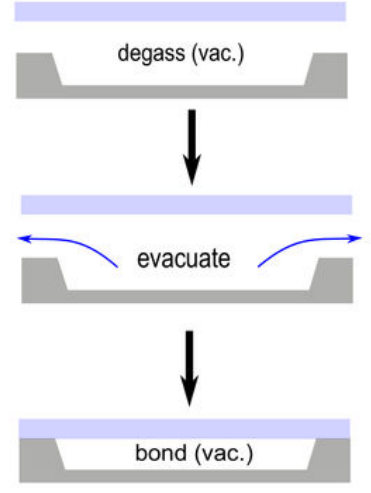

b)

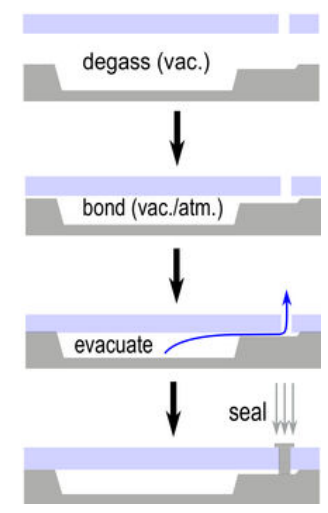

FIG. 4. Techniques to encapsulate a vacuum by evacuation before (a) and after (b) bonding.

thoroughly degas and evacuate chips and also allows the introduction of a novel structural scheme to drastically reduce leak rates discussed in Section V C.

The choice of materials from which the micro-MOT can be constructed is dependent on the sealing method and the typical process environments. Suitable materials for UHV have negligible vapour pressures, low outgassing and permeability rates, and are mechanically strong and machinable. Therefore metals such as stainless steel, aluminium, titanium and copper are predominant. The MicroMOT would require optical access for the cooling beams and fluorescence detection and so the chip must include an optically transparent section using a glass or glass-ceramic. Unfortunately there is a mismatch in the coefficients of thermal expansion (CTE) between glass and metal components which limit their baking, bonding, and operating temperatures. The exceptions are low expansion alloys such as Kovar and Invar, the former being specifically developed for glass-metal seals. Any remaining CTE mismatch can typically be alleviated with specially shaped sealing edges [136]. Nevertheless, we find that the majority of metals [137] do not have the extremely low outgassing rates [138, 139] highlighted in Table III, and their glass-metal seals [136] may not be adequately hermetic [140], or are impractical to implement into the microfabrication process (i.e. very high temperatures for extended periods). Alternative bonding methods are discussed in Section V C.

The MicroMOTs are likely to incorporate atom chips which commonly use silicon as a substrate due to its high thermal conductivity and the vast array of available semiconductor processing techniques [31]. Coincidentally silicon, as we shall see in the following sections, is a very suitable UHV material: it has extremely low permeation and outgassing rates at room temperature, it has several CTE-matched optical materials available, it is produced with a high purity (to the $9 \mathrm{~N}$ level), and can withstand high temperatures necessary for baking and bonding [141]. The disadvantage of this material is its brittle- 
ness [142], so only small structures can be fabricated using specialized techniques which is acceptable in our application, but not for large scale vacuum systems. Several well studied processes exist to clean silicon wafers and the lowest leak rates we have found for sealing technologies have been found for silicon-glass bonding (Section VC). It is also interesting to note that polysilicon coatings are commercially available to reduce the outgassing rates of stainless steel chambers [143]. Another advantage is that silicon is completely non-magnetic, which is important for manipulating atoms, and is a poor electrical conductor which reduces the deleterious effect of eddy currents during fast magnetic field switching [144] - a common issue with MOTs. Hence, in the following section we assume the chips are predominantly constructed from silicon and glass, with additional metal films for reflectors and getters.

\section{A. Pumping}

One cannot maintain a high vacuum without any form of pumping because no seal is perfect, all materials outgas to some extent, and no material is impermeable to all gases. However, by reducing the above effects as much as possible one can sustain vacuum with minimal pumping, especially in small volumes. A series of pumps are typically required to obtain UHV conditions, with roughing pumps achieving millibar pressures, turbo-molecular pumps down to to $10^{-8} \mathrm{mbar}$, and ion and sublimation pumps to finally reach $10^{-10}$ mbar and below. Once this is achieved the chamber is permanently sealed leaving only the latter two pumps to remove outgassing and permeated species. The roughing and turbo-molecular pumps no longer have any role in maintaining the vacuum and are usually removed. Operating an unsealed system is possible but should be avoided due to unnecessary power consumption and limitations on the ultimate vacuum due to 'backstreaming' and compression ratios of the pumps. If the commercial interest in cold atom technology required mass-production, the development and integration of sub-UHV pumps would be uneconomical due to their limited operating range. A more economical, and simpler, route would be to obtain UHV in a larger encapsulation chamber where several devices can be sealed leaving the need for only on-chip UHV maintenance pumps. In situations where the chip is regularly opened to atmosphere there exist several technologies, instigated in part by the DARPA 'Chip-Scale Vacuum Micro Pumps' program which aimed at obtaining $10^{-6}$ mbar with coin-sized pumps. This has resulted in impressively miniature displacement [145, 146], Knudsen [147], turbomolecular [148] and ion pumps [149-151]. Laser and Santiago [152] and Górecka-Drzazga [153] presented reviews of micropump developments previous to the DARPA programme. We shall see in Section VD that it is possible to construct chambers with extremely low outgassing materials such that minimal pumping is required. Ion pumps and getter pumps are good candidates for this role with the latter being more attractive due to their passive nature. Ion pumps are necessary in situations where ungettered species such as noble gases exist in the system. We aim to circumvent this issue by using impermeable windows and throughly degassed components.

Passive pumping elements take the form of getters which are metals, or alloys, that chemisorb typical gases found in high vacuum, namely $\mathrm{O}_{2}, \mathrm{CO}, \mathrm{N}_{2}$, and $\mathrm{H}_{2}$. Getters generally come in two varieties [154]: Evaporable getters are metals which are heated until their increased vapour pressure causes them to deposit on surrounding surfaces. This traps residual gases under the deposited layers, but the new surface also acts as a pump to impinging gases through chemisorption. Non-Evaporable Getters (NEGs) also chemisorb gases onto their surface, but in addition they absorb the reacted surface material into the getter bulk during heating (known as activation). Both types keep pumping gases at room temperature, albeit at a reduced rate, providing their surfaces are not saturated. NEGs activate at temperatures ranging around $200-800^{\circ} \mathrm{C}$, depending on their composition, whereas evaporable getters need to be heated to well above $700^{\circ} \mathrm{C}$. Therefore the choice of NEGs for our MicroMOT is obvious, and is further validated by its adoption in the MEMs vacuum encapsulation industry, however we shall also discuss recent advances in Group I/II evaporable (or reactive) getters.

Non-evaporable getters are made of Group IV/V metals and alloys, such as $\mathrm{Ti}, \mathrm{Zr}, \mathrm{V}$, Hf etc, and may also include metals such as $\mathrm{Al}$ and $\mathrm{Fe}$. These are elements with high oxygen solubility, high diffusivity, and high enthalpy of adsorption for many gases found in vacuum [155]. When exposed to air, the surface of the NEG quickly passivates forming oxides, nitrides and carbides in an 2-3 nm layer [156]. Heating the NEG in vacuum, known as activation, causes these compounds to diffuse into the bulk leaving a fresh metallic surface pump. Typical oxygen solubilities for NEG compounds are on the order of $10 \%$, so a $1 \mu \mathrm{m}$ thin film can undergo $\sim 100$ reactivation cycles after air exposure, however the pumping efficiency begins to reduce after a few cycles [157]. This corresponds to an approximate total capacity of $10^{12}$ molecules per cubic centimetre. Recent in-situ studies of NEG activation with individual gases at temperatures above activation indicate far higher capacities of the order of $10^{5}$ monolayers of carbon monoxide [158] due to the increasingly uniform oxygen concentration in the film with temperature. Hydrogen diffuses readily in the bulk, and so the capacity is approximately two orders of magnitude higher than surface pumped species at room temperature. Embrittlement of the film at very high hydrogen concentrations (above 1\%) can result in delamination and so should be avoided [159]. When NEGs are deposited as thin films they also act as outgassing barriers [160], thus turning gas sources into pumps and greatly reducing the ultimate pressure. Noble gases and some hydrocarbons, 
such as methane, are not pumped by NEGs at room temperature. Therefore the MicroMOT will need to be sealed at UHV to ensure the majority of gases, especially the nobles, are evacuated prior to encapsulation.

The bonding techniques discussed in Section V C require temperatures up to $400^{\circ} \mathrm{C}$, which will lead to increased outgassing (see Section VD) and a reduction of the NEG lifetime due to saturation. Moreover, reactivation of the getter to rejuvenate its pumping rate during the sealed devices' lifetime will lead to increased outgassing, permeation and possibly leakage. Therefore it is prudent to use an NEG alloy with a very low activation temperature, and high pumping rate and capacity. The beam lines of particle accelerators require XHV environments, and their very large volume presents an issue for efficient and uniform pumping. Several decades of research at CERN have been devoted to finding NEGs both which activate during the chamber baking procedure $\left(\sim 250^{\circ} \mathrm{C}\right)$ and can also coat all internal surfaces [161]. Their findings have shown that sputtered Ti-Zr-V alloys of nearly equal ratios can be activated at $180^{\circ} \mathrm{C}$, and using these coatings, together with ion pumps, they have demonstrated the lowest room temperature vacuum of $10^{-14}$ mbar [162]. The pumping rate of NEG films depends on their surface area and so the CERN team have also looked into the effects of substrate and deposition parameters to increase pumping rates and capacities [163]. Additional attractive properties of Ti-Zr-V NEG films are their high adhesion, thermal and vibration stability, resilience to standard wafer cleaning processes, and commercial availability [164]. Table IV shows the typical pumping rates and capacities of Ti-Zr-V NEG thin films whose values will be used in the following sections. Not all gases are pumped equally and some lead to reduced pumping speed of the NEG at high surface coverage (for example, $\mathrm{CO}$ reduces the pumping rate and capacity of $\mathrm{H}_{2}$ and $\mathrm{N}_{2}$ ) which are shown in Table IV. This effect must be accounted for when calculating the lifetime of getter pumped devices. The unintentional incorporation of noble gases in sputtered films can result in outgassing which may endanger the vacuum [165, 166] and this will be discussed in Section VD. As a result, alternative methods, such as vacuum arc deposition [156] or e-beam evaporation [167], should be considered.

Most gases only chemisorb on the NEG surface and show negligible pumping after a monolayer is formed. Hydrogen is the exception as it diffuses throughout the entire bulk of the getter and so only the thickness of the film defines the capacity. There exists a thermal equilibrium between the absorption and desorption of hydrogen from the NEG. This is dependent on the hydrogen concentration [168] and thus can be used to predict the residual pressure in our devices. This value, known as the disassociation pressure, follows Sieverts' law and has been measured for Ti-Zr-V films [157]. It was found to be given by:

$$
\log _{10}\left(P_{H_{2}}\right)=2 \log _{10}\left(x_{H}\right)+14.324-\frac{8468}{T}
$$

Where $P_{H_{2}}$ is in millibar, $x_{H}$ is the fraction of hydrogen in the film and $T$ is the temperature in kelvin. We can see that for a very saturated film $\left(x_{H}=0.01\right)$ the pressure is negligible at room temperature $\left(10^{-19} \mathrm{mbar}\right)$ and only endangers the vacuum at temperatures above $150^{\circ} \mathrm{C}$, at which point helium permeation through the glass wafer becomes equally problematic, as we shall see in Section VB.

Earlier we dismissed evaporable getters on the basis of high operating temperatures and vapour phase gases. There is a new type of evaporable, or more specifically reactive, getter [169] being investigated which uses Group I/II atoms, specifically lithium, as the pumping medium [170-173]. Early studies of alkali metal dispensers showed that they improve the pumping rate of the system [105], and barium is the original and highly effective getter material widely used in cathode ray tubes. Reactive getters will react and bind strongly to the common residual gases found at UHV and therefore our devices may experience an improvement of the vacuum during operation. Experiments have shown that the pumping rate for carbon monoxide by lithium getter films is similar to Ti-Zr-V NEGs but with a capacity over $10^{4}$ times greater. This would be very advantageous to remove outgassed species during bonding which could otherwise saturate Ti-Zr-V films. Most of the work on these reactive getters has focused on lithium due to its low vapour pressure and ability to form stable compounds with a number of gases. We suspect rubidium will provide some gettering, but not to the extent of lithium due to the former's high vapour pressure. For example, the disassociation pressure [170] of $\mathrm{LiH}$ at $300 \mathrm{~K}$ is $10^{-21}$ mbar whereas that of $\mathrm{RbH}$ is $10^{-7}$ mbar, only marginally less than rubidium vapour pressure [89, 174]. It is expected, however, that pumping of oxygen and carbon monoxide will be more effective. With its greater capacity and ability to pump additional gases such as methane, which NEGs cannot, a lithium getter could replace, or complement, the NEG in the MicroMOT if the necessary vacuum cannot be pumped by NEGs alone [175].

TABLE IV. Typical pumping rates, sticking factors and capacities of Ti-Zr-V NEGs at room temperature for a $1 \mu \mathrm{m}$ film. The values in parentheses indicate the pumping rate after carbon monoxide saturation [157]. The capacities can be increased by nearly an order of magnitude by heating the substrate during deposition to increase surface area.

\begin{tabular}{lccc}
\hline Gas & $\begin{array}{c}\text { Sticking } \\
\text { factor }\end{array}$ & $\begin{array}{c}\text { Pumping } \\
\text { rate }\left(1 \mathrm{~s}^{-1} \mathrm{~cm}^{-2}\right)\end{array}$ & $\begin{array}{c}\text { Capacity } \\
\left(\mathrm{cm}^{-2}\right)\end{array}$ \\
\hline $\mathrm{H}_{2}$ & $8 \times 10^{-3}$ & $0.35(0.1)$ & $>10^{16}$ \\
$\mathrm{~N}_{2}$ & $1.5 \times 10^{-2}$ & $0.17(0.1)$ & $1.5 \times 10^{14}$ \\
$\mathrm{CO}$ & 0.7 & 8 & $10^{15}$ \\
\hline
\end{tabular}


In the following sections we assume:

- An internal volume of $0.5 \mathrm{~cm}^{3}$.

- A surface area of $5 \mathrm{~cm}^{2}$.

- An NEG area of $1 \mathrm{~cm}^{2}$ with a thickness of $1 \mu \mathrm{m}$.

\section{B. Permeation}

No material is absolutely impermeable to all gases, and so it is inevitable that they will diffuse through the walls of any chamber and finally desorb into the vacuum. We shall address the issue of permeation before that of outgassing and leaks as this is seen by many as the limiting factor in achieving UHV in small, sealed, well-degassed volumes. Permeation is complex and requires several processes to become a vacuum risk: 1) The gas molecules in the external environment impinge on the outer surface of the chamber wall and physisorbed, 2) they disassociate if the surface enthalpy is greater than their bonds, 3) they are absorbed under the surface layer and diffuse through the bulk along the concentration gradient (Fick's law), 4) the gas atoms must then overcome any surface energy barriers, and 5) desorb from the surface directly or recombine with other ions to desorb as a molecule. These processes strongly depend on the type of permeating gas and the chamber wall material. For example, noble gases will permeate glasses, but not most metals due to the latter's more crystalline structure and weak surface interaction. As noble gases are not pumped by NEGs their permeation is of greatest importance and we shall devote the majority of this section to them, however we will also discuss the effect of hydrogen as this is the second fastest permeating gas (see Table VI).

Surface effects, which will be discussed in Section VD, tend to reduce the permeation rate so the simple process of bulk diffusion can be considered the limiting factor of permeation. The amount of gas flowing diffusively across a membrane of area $A$ and thickness $d$ between two regions of pressure $P_{\text {ext }}$ and $P_{\text {int }}$ is

$$
\frac{d Q}{d t}=\frac{K A\left(P_{e x t}-P_{i n t}\right)}{d}
$$

where $K=D S$ is the permeation rate $\left(\mathrm{cm}^{2} \mathrm{~s}^{-1}\right), D$ is the diffusion constant $\left(\mathrm{cm}^{2} \mathrm{~s}^{-1}\right)$ and $S$ is the solubility $\left(\mathrm{cm}^{3}(\mathrm{STP}) / \mathrm{cm}^{3}\right)$. Both $D$ and $S$ typically follow an Arrhenius-type temperature dependence and the former is quoted in the literature as

$$
D(T)=D_{0} \exp \left(\frac{-E_{D}}{k_{B} T}\right)
$$

where $E_{D}$ is the diffusion energy, $k_{B}$ is the Boltzmann constant and $T$ is the temperature. The variation of $S$ can be much more complicated [176], however over a limited range of temperatures the Arrhenius form is adequate, and for most materials $S$ does not change appreciably compared to $D$ with temperature. Values of diffusion, solubility and permeability of the materials highlighted in this study can be found compared to the common vacuum materials, stainless steel and Pyrex, in Table V. Equation 2 assumes the gas is already fully dissolved in the membrane, which is not the case in thoroughly degassed materials. Therefore there is a period of time before the gas will 'break through' to the evacuated volume, and in materials with very low diffusion rates this can be extremely long [177]. The pressure increase of a cavity of volume $V$ at a time $t$ due to a gas permeating through a degassed membrane is [178]:

$$
P_{c}=\frac{A D S P_{e x t}}{V d}\left[t-\frac{d^{2}}{6 D}-\frac{2 d^{2}}{\pi^{2} D} \sum_{m=1}^{\infty} \frac{(-1)^{m} e^{\frac{-m^{2} \pi^{2} D t}{d^{2}}}}{m^{2}}\right]
$$

Since the micro-MOT chambers will require at least one optically transparent viewport the permeation of helium through glass will be a significant issue. The amorphous network structure of glass forming oxides provide channels for helium to diffuse. Not all glasses are alike however, and the addition of 'modifier' molecules can act to plug the holes in the network, resulting in very low permeation rates [179]. We aim to seal glass to silicon and therefore must match CTEs to reduce stresses and therefore increase yield. Pyrex is the most common glass bonded to silicon due to their comparable CTEs and its sodium content required for anodic bonding [181] (see Section VC). This borosilicate glass is so highly permeable to helium that we should expect to lose UHV several days after bonding (see Figure 5). Much work was carried out in the 1960s and 1970s on the permeation

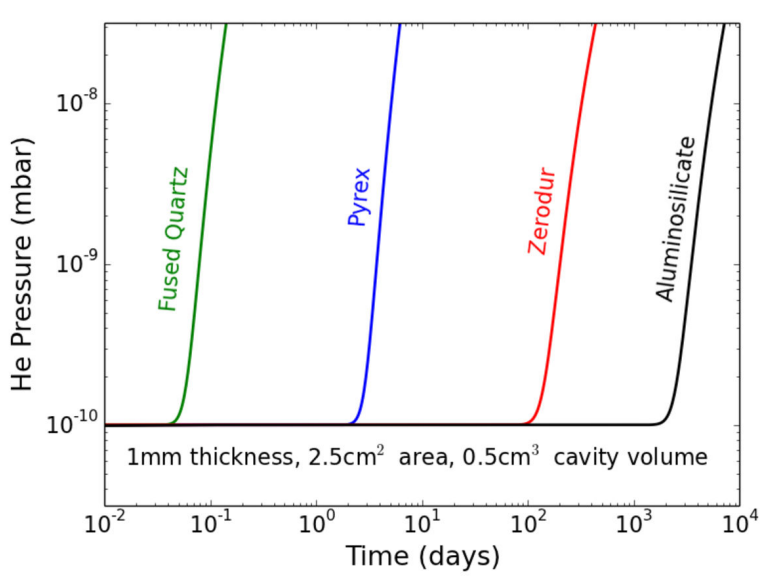

FIG. 5. The permeation rate of various $1 \mathrm{~mm}$ optical wafers exposed to atmospheric helium after initial evacuation to $10^{-10}$ mbar after complete degassing. We have calculated the values using Equation 4 and used the data for Corning 1720 to define the properties of AS glass [179, 180]. 
rates of gases through glass, and the results showed that Alumino-Silicate (AS) glass, those with approximately $20 \% \mathrm{Al}_{2} \mathrm{O}_{3}$ or more composition, had permeation rates five orders of magnitude lower than Pyrex [179, 182]. Figure 5 shows a comparison of the permeation rates of helium through AS glass, Pyrex, fused silica, and Schott Zerodur glass-ceramic, calculated using Equation 4. This latter material is commonly used in UHV systems requiring low permeation and its very low CTE makes it well suited for bonding. We can also see that Corning 1720 series AS glass is more than capable of maintaining vacuum for our target time. Its CTE is well matched with silicon to which is can be anodically bonded, albeit at much higher temperatures than Pyrex due to the low alkali content [183-185].

Other AS glasses are commercially available, but too numerous to list here, and we mention that load-borate and soda-lime glasses also have low permeability. Silicon carbide [186] has a very low permeability [187], can be bonded to silicon [188], and has been used for atom chips due to its transparency and high thermal conductivity [189]. Sapphire and glass-ceramics such as Spinel [190] are likely to have extremely low permeation rates, but yet again are not well CTE matched. Hard crystalline optical coatings may also reduce permeation. Graphene, amongst its many other attractive properties, has shown a permeability rate equal to bulk Pyrex, yet requiring only a single monolayer [191]. Unfortunately uniform coatings over large areas are as yet unavailable, but graphene-oxide may be a suitable alternative [192].

Norton [199] measured the permeation of other gases through fused silica, as shown in Table VI, and we can see that, in general, larger molecules have lower permeation rates but can depend on surface interactions and solubilities (compare $\mathrm{H}_{2}$ and $\mathrm{Ne}$ ). Hydrogen diffuses through glass as a molecule $[176,197]$ and so, as shown in Table $\mathrm{V}$, it will have a far lower permeation rate than helium. Coupled with an order of magnitude lower atmospheric partial pressure compared to helium, and the ability to pump the gas with NEGs, hydrogen permeation can be neglected. Very little data is available on the hydrogen permeability through AS glass and so we have used a scaling law by Souers et al [195], the glass composition from Altemose [179], and the Pyrex solubility to calculate the diffusivity constants in Table V.

TABLE VI. Permeation of different gases [204, 205] through fused silica [199].

\begin{tabular}{lcc}
\hline Gas & $\begin{array}{c}\text { Relative permeation } \\
\text { rate at } 700^{\circ} \mathrm{C}\end{array}$ & $\begin{array}{c}\text { Van der Waals } \\
\text { radius, nm }\end{array}$ \\
\hline $\mathrm{He}$ & 1 & 0.133 \\
$\mathrm{H}_{2}$ & 0.1 & 0.15 \\
$\mathrm{Ne}$ & 0.02 & 0.141 \\
$\mathrm{Ar}$ & $<10^{-7}$ & 0.176 \\
\hline
\end{tabular}

We now address the second material in our system: silicon. Measurements of helium permeation through silicon extrapolated from high temperatures show that, like metals, silicon is practically impermeable to all noble gases. Using measured values for solubility [193, 206] and typical atmospheric helium content, we should not expect to find a single atom within a cubic centimetre of silicon. We note that recent studies [207, 208] looking at the hermeticity of glass frit encapsulation and other bonding methods have indicated that helium permeation through silicon at room temperature may be more significant than expected. However, more work is required to confirm this against the large bulk of research into helium bubble formation in silicon which agrees with the original low permeation result [209]. Hydrogen, on the other hand, is known to permeate silicon albeit predominantly in atomic form at room temperature. This matter will be discussed in great detail in Section VD as the permeation rate is related to outgassing. The results in Table $\mathrm{V}$ show that the permeation rate of hydrogen through silicon at room temperature is negligible.

\section{Leaking}

No seal is perfect as the bonding of materials will inevitably lead to a route for gases to travel, via microchannels and defects, or merely a local variation in the permeation rate. Standard UHV systems predominantly use Conflat type seals which employ knife-edges to bite into OHFC copper gaskets and join metal components together, and 'housekeeper' type seals for glass-to-metal interfaces. Commercial vacuum products quote leak rates less than $10^{-11} \mathrm{mbar}^{-1 \mathrm{~s}^{-1}}$ (STP), usually limited by the resolution of the leak detector [210]. These types of seals are not suitable for wafer-level fabrication of vacuum encapsulated micro-electronics and so several new methods have been developed using chemically formed seals, or simply relying on the attraction between perfectly flat surfaces [211]. The small volume and long lifetime of many microelectronic chips may preclude the use of internal pumping mechanisms, meaning that the seals must have extremely low leakage, more so than those demanded by standard vacuum systems.

$$
Q_{L}=\frac{V \Delta P}{\Delta t}
$$

Using Equation 5 we can calculate the highest permissible leak rate for a gas at atmospheric pressure leaking into our specified volume of $V=0.5 \mathrm{~cm}^{3}$, such that the pressure does not rise by $50 \%\left(\Delta P=0.5 \times 10^{-10} \mathrm{mbar}\right)$ over $\Delta t=1000$ days, to be $3 \times 10^{-22}$ mbarls $^{-1}$. This rate seems unachievable, but we must consider that some gases are pumped away by the NEGs and so may permit a higher leak rate, and many gases have low atmospheric partial pressures. For example the partial pressure of helium in the atmosphere is $5 \times 10^{-3}$ mbar and so one can permit a leak rate [212] of $1.5 \times 10^{-17} \mathrm{mbar} \mathrm{s}^{-1}$. 
TABLE V. Bulk diffusivities and solubilities (at STP) of helium and hydrogen in silicon, Pyrex (Corning 7740), aluminosilicate (Corning 1720), and stainless steel. We have also tabulated the permeation rates at $20^{\circ} \mathrm{C}$ and $500^{\circ} \mathrm{C}$ to compare values more easily.

Silicon [193, 194] Aluminosilicate [179, 195, 196] Pyrex [176, 179, 195-198] Stainless steel [199, 200]

\begin{tabular}{|c|c|c|c|c|}
\hline $\begin{array}{l}\text { Helium }^{\mathrm{a}} \\
\mathrm{D}_{0}\left(\mathrm{~cm}^{2} \mathrm{~s}^{-1}\right)\end{array}$ & $5.2 \times 10^{-3}$ & $3.7 \times 10^{-4}$ & $4.6 \times 10^{-4}$ & Impermeable [199, 203] \\
\hline $\mathrm{E}_{D}(\mathrm{eV})$ & -0.82 & -0.52 & -0.28 & - \\
\hline $\mathrm{S}_{0}\left(\mathrm{~cm}^{3}(\mathrm{STP}) \mathrm{cm}^{-3}\right)$ & $2.8 \times 10^{-4}$ & 0.0016 & 0.005 & - \\
\hline $\mathrm{E}_{S}(\mathrm{eV})$ & -0.77 & - & - & - \\
\hline $\mathrm{K}\left(\mathrm{cm}^{2} \mathrm{~s}^{-1}\right) @ 20^{\circ} \mathrm{C}$ & $3.2 \times 10^{-34}$ & $6.8 \times 10^{-16}$ & $3.5 \times 10^{-11}$ & - \\
\hline @ $500^{\circ} \mathrm{C}$ & $4.8 \times 10^{-17}$ & $2.4 \times 10^{-10}$ & $3.4 \times 10^{-8}$ & - \\
\hline Hydrogen $^{\mathrm{b}} \mathrm{c}$ & & & & \\
\hline $\mathrm{D}_{0}\left(\mathrm{~cm}^{2} \mathrm{~s}^{-1}\right)$ & $9.7 \times 10^{-3}$ & $2.08 \times 10^{-7} \mathrm{~K}^{-1}$ & $1.4 \times 10^{-5}$ & $1.2 \times 10^{-2}$ \\
\hline $\mathrm{E}_{D}(\mathrm{eV})$ & -0.48 & -0.67 & -0.24 & -0.56 \\
\hline $\mathrm{S}_{0}\left(\mathrm{~cm}^{3}(\mathrm{STP}) \mathrm{cm}^{-3}\right)$ & 90.4 & 0.038 & 0.038 & 0.3 \\
\hline $\mathrm{E}_{S}(\mathrm{eV})$ & -1.86 & -0.12 & -0.12 & -0.11 \\
\hline $\mathrm{K}\left(\mathrm{cm}^{2} \mathrm{~s}^{-1}\right) @ 20^{\circ} \mathrm{C}$ & $5.7 \times 10^{-40}$ & $6.1 \times 10^{-23}$ & $3.4 \times 10^{-16}$ & $1.0 \times 10^{-14}$ \\
\hline @ $500^{\circ} \mathrm{C}$ & $1.2 \times 10^{-15}$ & $4.3 \times 10^{-14}$ & $2.4 \times 10^{-12}$ & $1.4 \times 10^{-7}$ \\
\hline
\end{tabular}

a We are unable to find helium solubility in silicon data other than van Wieringen et al [193] which is somewhat unreliable due to the limited measurement range. In the tabulated values we have used that data with the theoretical energy of solution [194] to calculate the prefactor. The diffusivity values are also theoretical but agree with experimental results [193, 201, 202].

b We have assumed the same solubility for AS glass as for Pyrex due to the small variation found between glasses and the minimal temperature variation[197, 198].

${ }^{c}$ Like helium, the commonly quoted values measured by van Wieringen et al [193] are extrapolated from a narrow high temperature range and can be assumed to indicate the highest diffusion rate (see Section VD).

Reactive gas leakage is limited by the pump rate and capacity of the NEG films. In light of this we may model the lifetime of the device due to NEG saturation using the following formula:

$$
\frac{d P_{c}}{d t}=\frac{1}{V}\left(Q_{L}-L_{P} P_{c}(1-\theta)^{k}\right)
$$

Where $P_{c}$ is the internal pressure (mbar), $Q_{L}$ is the leak rate $\left(\mathrm{mbarls}^{-1}\right), L_{P}$ is the pumping rate of the getter $\left(\mathrm{ls}^{-1}\right), \theta$ is the fractional surface coverage, and $k$ is the order of desorption. The effect of surface coverage on pumping depends on the gas/surface chemistry, temperature, and surface geometry (flat, granular, etc.). This topic is too extensive to detail here but can be found in most surface science graduate texts [213]. We find that the Langmuir adsorption isotherm, $(1-\theta)^{k}$, models the majority of data on NEG pumping rates [157] adequately for our purposes due to the low surface coverage. The pressure inside the cavity will drop to base value:

$$
P_{\text {base }}=\frac{Q_{L}}{L_{p}}
$$

As noted earlier, the effect of saturation by some gases (for example carbon monoxide) reduces the pumping speed of other gases [157] which we do not take into account dynamically in the model, but assume the lowest pumping speed as the 'worst case scenario'.

There is a sharp drop in pumping speed at saturation allowing us to simplify the lifetime calculation. By assuming that the pumping rate is constant until the NEG

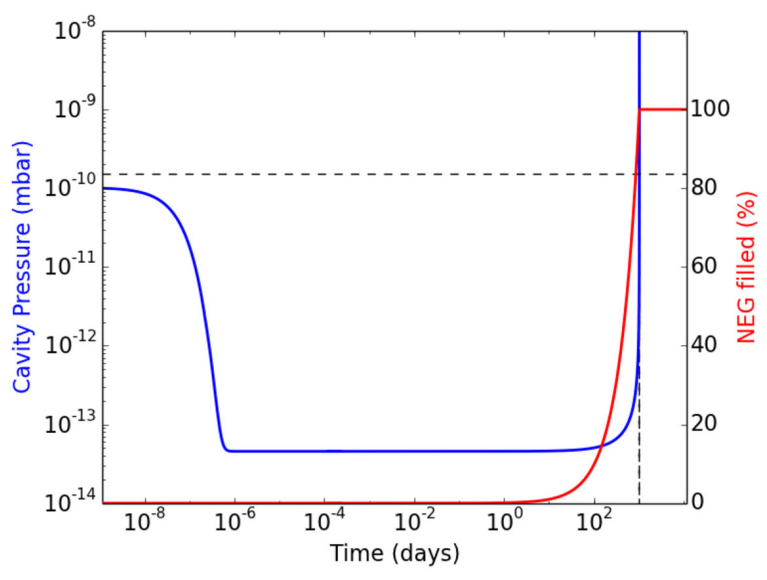

FIG. 6. The internal pressure (blue) and NEG coverage (red) with an atmospheric leak of nitrogen at a rate $6.5 \times 10^{-15} \mathrm{mbarl}^{-1}$ (air) calculated using Equation 6. The horizontal dashed line indicates the target pressure and the vertical line the result of Equation 8. The cavity is initially at $10^{-10}$ mbar.

has reached its capacity of $C_{G}$ (moles), at which point it ubruptly drops to zero $(\theta=1)$, and substituting the ideal gas formula into Equation 6, we find an approximate value for the maximum permissible leak rate:

$$
Q_{L}(\max )=\frac{C_{G} R T}{\tau_{L}}
$$


where $R$ is the ideal gas constant, $T(\mathrm{~K})$ is the temperature and $\tau_{L}(\mathrm{sec})$ is the lifetime. If we assume a pumping speed for nitrogen, the most abundant atmospheric gas, of $0.1 \mathrm{ls}^{-1}$, a capacity of $10^{14}$ molecules, with a lifetime of 1000 days: the maximum permissible leak rate is $5 \times 10^{-15} \mathrm{mbarls}^{-1}$ with $P_{\text {base }}=5 \times 10^{-14}$ mbar. Note that Equation 8 is independent of both the pumping rate and background pressure because it assumes them to be at equilibrium, therefore one must use this equation alongside Equation 7 to ensure the correct base pressure. Figure 6 compares the numerical solution of Equation 6 with the approximate value from Equation 8 and we find perfect agreement. We note that the capacity is that for a single monolayer and so the lifetime can simply be extended through reactivation cycles. Hydrogen diffuses into the bulk and so reactivation does not increase the getter lifetime, but the NEG capacity for hydrogen can be two to three orders of magnitude greater (see Table IV), so is not as much of a concern.

In Section V we discussed the methods to encapsulate microfabricated vacuum devices and here we shall explore the details of suitable bonding methods and their quality. We foresee the need for at least two bonding processes: one to bond the 'atom chip' to the structure wafer (silicon to silicon, or gold to silicon), and another to bond the glass capping wafer to the structure layer (glass to silicon). As many bonding technologies exist we will only consider those which are well established, have demon-

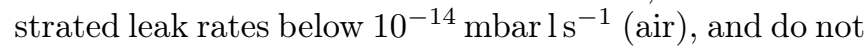
require temperatures above $400^{\circ} \mathrm{C}$ so as to reduce outgassing, stress, and protect chip components. We have not included indium bonding (or alloys thereof) as indium melts below the activation temperature of NEGs and therefore requires specialized surface geometries and processes [134] to reduce outgassing and ensure good seals. Also, indium bonding can require several additional films to improve surface wetting, and the leak rate may not sufficiently low [214].

\section{Glass Frit}

Glass frit bonding is a well established technique which involves the deposition of a low melting point glass compound between two materials. The glass is heated first to outgas the organic binder compounds, and is then raised to the glass transition temperature which melts and seals the two surfaces upon cooling. The vacuum hermeticity of this technique has been explored extensively by Sparks et al [215], but no absolute leaks rates have been quoted. We believe glass frit bonding to have a leak rate below $10^{-15} \mathrm{mbar}^{1 \mathrm{~s}^{-1}}$ by considering the lifetime, internal volume, and pressure inferred by the integrated resonator's Q-factor [216]. However, the pressure measured $\left(\sim 10^{-3}\right.$ mbar $)$ is at the limit of the gauging technique and one does not know the residual pressure immediately after bonding. Possible issues may include insufficient degassing of the organic binder materials, limitations on pre-baking temperatures, incompatible CTEs (although they can be engineered to match the application) and the need for additional materials. Of the four bonding methods presented here, glass frit has the least sensitivity to surface quality and can be used to seal electrical feedthroughs.

\section{Eutectic Bonding}

A eutectic alloy is one where the melting point of the constituent materials is lowered on contact. For example gold and silicon individually have melting points above $1000^{\circ} \mathrm{C}$, but when they are pressed together they will melt at $363^{\circ} \mathrm{C}$ at their interface $[217,218]$. Subsequent cooling will form an alloy with high hermeticity and a strong bond. Other suitable eutectic alloys exist, such as gold and tin [219], but we highlight the gold-silicon system as many atoms chips employ gold as a reflector and conductor. This bond has demonstrated the lowest leak rate that we have found [132], below $10^{-15} \mathrm{mbarls}^{-1}$. Possible disadvantages include the need for multilayer films to prevent interlayer diffusion [218], the requirement of inert gas storage before bonding to prevent the native oxide growth on silicon, and the temperature restrictions post bonding, as further heating remelts the alloy and degrades the bond [220].

\section{Anodic Bonding}

Anodic bonding occurs between an oxide forming metal (or semiconductor) and an alkali containing glass, by heating the two materials together $\left(300-500^{\circ} \mathrm{C}\right)$ with the simultaneous application of a high voltage (100$1000 \mathrm{~V})$ across the interface[181, 183]. The mobility of the alakli ions (typically sodium or lithium) in the glass is increased with temperature and they are pulled away from the interface by the electric potential. The residual non-bridging oxygen atoms at the interface then bond with the silicon. The high electric potential gradient has the additional effect of pulling the two surfaces into intimate contact which overcomes surface inhomogeneities. The two materials (usually silicon and borosilicate glass) must have very flat surfaces, below $10 \mathrm{~nm}$, and be CTE matched to avoid stress fractures during cooling. Hermeticity measurements show that the leak rate is below $10^{-14} \mathrm{mbarls}^{-1}$, with few residual gases other than oxygen, which is produced during bonding at the inner seam. This residual gas source can be significant, especially in small evacuated volumes, and so getter films are mandatory (see Section VD). The leak rate measurements [221, 222] included the effect of the bonding area around the cavity and found no variation, from which we infer that the seal is absolutely hermetic and possibly limited only by permeation. 


\section{Direct Bonding}

Direct bonding is the result of the attractive Van der Walls forces between atomically flat surfaces. Semiconductor and glass wafers are routinely produced with the required flatness and this technique requires no additional materials, does not release gases (unlike anodic and frit bonding), and is hermetic [222] (Leak rates below $\left.10^{-14} \mathrm{mbar} \mathrm{l} \mathrm{s}^{-1}\right)$. Unfortunately, for the silicon-silicon direct bond, very high post-annealing temperatures (up to $1000^{\circ} \mathrm{C}$ ) are required to ensure a high bonding yield and to reduce voids. This latter effect is due to gases desorbing between the surfaces which becomes trapped. Bonding in vacuum produces fewer voids, due to a thorough degassing before sealing, to the point that strong bonds form at room temperature without the need for post annealing [223]. The extremely flat surfaces are difficult to retain during processing and even sub-micron particles will result in debonding. CTE-matched glasssilicon direct bonding occurs with lower annealing temperatures [224], and the glass can absorb gases released between the interface.

To summarize the bonding techniques, there are several methods to obtain reliable leak rates lower than

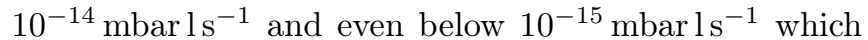
are required to maintain UHV. Anodic and direct bonds may even be absolutely hermetic, limited by permeation, but their absolute leak rates were beyond the sensitivity of their measurements. Eutectic bonding has shown the lowest measured leak rates and is a reliable and low outgassing method. Glass frit may also have equally low leak rates, but the residual gas pressure released during bonding is unknown.

The atmosphere contains several noble gases [132] including argon (9.3 mbar), neon $\left(1.8 \times 10^{-2} \mathrm{mbar}\right)$, and helium $\left(5 \times 10^{-3} \mathrm{mbar}\right)$, where the values in parentheses are the atmospheric partial pressures. Their leak rates are proportional to $(T / M)^{0.5}$, where $T$ is the temperature in kelvin and $M$ is their mass, hence helium leaks at the fastest rate and is often used in hermeticity tests [225] known as 'Helium Bombing'. If we assume a leak rate which is proportional to the pressure differential across the bond, the effect of argon, due to its relatively high atmospheric partial pressure will be most significant. The maximum permissible leak rate for argon is $1 \times 10^{-19} \mathrm{mbar} \mathrm{s}^{-1}$. The significance of this gas has been mentioned in the literature [226], but is generally ignored as helium permeation through glass is considered to be a more pressing issue. If we compare the lowest measured leak rate for the bonding methods of $10^{-15} \mathrm{mbarls}^{-1}$ (air) we find all the noble gases endanger UHV, however helium and neon leakage need only be reduced by factor of 15 and 25, respectively, which may be possible with thicker bonding seams or external barrier coatings, whereas argon must be reduced by nearly $10^{4}$.

There is, however, a very simple scheme to reduce the leak rate by several orders of magnitude: Simply by placing the vacuum chamber inside another. This can be achieved practically by introducing a buffer cavity, or moat, within the seam such that the slow leakage into the moat results in an even slower leakage into the main vacuum cavity [227]. This can be modeled by the following formulae and solved numerically:

$$
\begin{gathered}
\frac{d P_{b}}{d t}=\frac{1}{V_{b}}\left(C_{a b}\left(P_{a}-P_{b}\right)-C_{b c}\left(P_{b}-P_{c}\right)\right) \\
\frac{d P_{c}}{d t}=\frac{C_{b c}\left(P_{b}-P_{c}\right)}{V_{c}}
\end{gathered}
$$

where $P_{i}$ is the pressure, $V_{i}$ is the volume, and $C_{i j}$ is the conductance between $i$, and $j$, in which the subscripts $i, j=a, b, c$ refer to the air, buffer, and cavity, respectively. We have independently modelled this effect and found stark, but advantageous, differences from the original study by Gan et al [227]. We suspect that an error was made in tabulating their results, which also clarifies their unexplained lifetime increase for 100 mbar cavities. If we assume a main cavity volume of $0.5 \mathrm{~cm}^{3}$ and a moat volume of $0.05 \mathrm{~cm}^{3}$ we can reduce the leakage rate by factor of $2 \times 10^{5}$ as shown in Figure 7 . This allows us to use bonds with leak rates in the range of $10^{-14}$ mbar $1 \mathrm{~s}^{-1}$ (air) which is technically feasible with all the bonding techniques considered earlier, and also reduces the NEG limitations on reactive gases. The moat does not have to be bonded at UHV as the model shows very little variation below an initial moat pressure of $10^{-6}$ mbar.

\section{Outgassing}

Outgassing is the release of gas from a material's surface and bulk. We distinguish this from permeation in that the latter assumes a completely degassed material, whereas outgassing is the result of gases that are difficult to remove from the bulk. These have entered either from diffusion during storage, processing, or from the production of the material itself. Cleanliness is of utmost importance in achieving UHV and we assume all components have been through standard wafer cleaning processes, such as an RCA and ozone plasma. This latter technique has also been shown to improve bond strengths and reduce temperatures direct and anodic bonding [228, 229]. We shall not go into further detail regarding cleaning here and direct the interested reader to the references [230-233]. We do highlight that detergents were found to remove vanadium from the NEG films and so should be avoided [234].

Outgassing is the largest source of gas in well-sealed UHV systems and so usually defines the lowest base pressure for a specific pumping rate $L_{p}$ according to Equation 7. Hydrogen is the dominant gas at UHV, for 


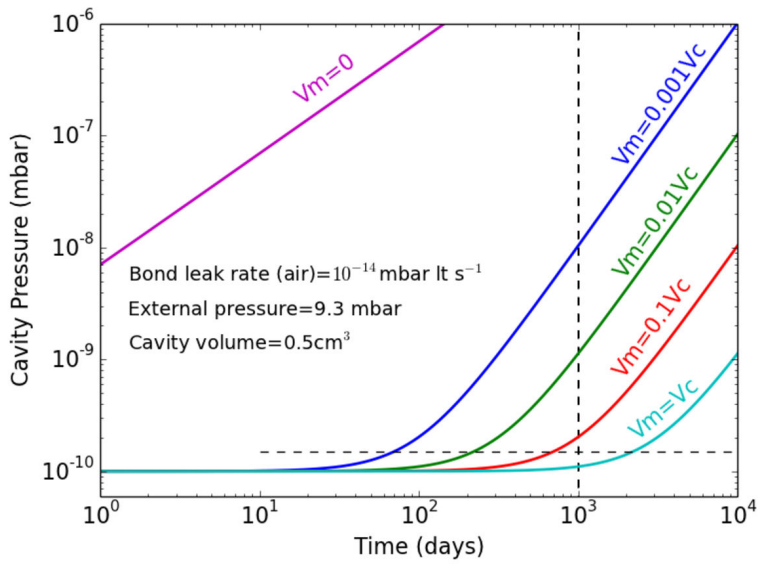

FIG. 7. Numerical solution of Equations 9 for various ratios of moat/cavity volume. We assume argon is leaking from the atmosphere, and the quoted leak rate is for each bond seam (airto-moat and moat-to-cavity) adjusted to atmospheric pressure to compare with literature values. The horizontal dotted line represents a $50 \%$ rise in pressure from an initial value of $10^{-10} \mathrm{~Pa}$, and the vertical line indicates 1000 days. In this simulation $V_{m}=0.25 V_{c}$ would meet the target property with an overall leak rate of $6 \times 10^{-21} \mathrm{mbar} \mathrm{m}^{3} \mathrm{~s}^{-1}$ (air).

which the NEGs have a pumping rate on the order of $0.1 \mathrm{ls}^{-1} \mathrm{~cm}^{-2}$, and so to achieve $10^{-10}$ mbar one must ensure the outgassing rate is below $10^{-11} \mathrm{mbarl} \mathrm{s}^{-1} \mathrm{~cm}^{-2}$. This is greater than NEG capacity-limited outgassing rate calculated using Equation 8 and so the base pressure will not be limited by the pumping rate [235].

There is no standard model for predicting the outgassing properties of all materials as many different mechanisms are involved [236, 237] but can be essentially split into two sources: surface and bulk. We assume that the surfaces are clean in that common contaminates such as organics have been thoroughly removed leaving only atmospheric and some processing species, namely water, hydrogen, carbon monoxide, and noble gases. We can calculate the surface desorption rate using:

$$
\frac{d N}{d t}=\frac{N \theta^{k}}{\tau_{s}} \exp \left(\frac{-E_{S}}{k_{B} T}\right)
$$

where $N$ is the surface density (molecules $\mathrm{cm}^{-2}$ ), $\theta$ is the fractional surface coverage, $k$ is the desorption order, $\tau_{s}$ is the sojourn time (typically $10^{-13} \mathrm{~s}$ ), and $E_{S}$ is the desorption energy [238]. Outgassing from the surface usually occurs at the fastest rate as physisorbed, or weakly chemisorbed gases, have low desorption energies. Strongly bound molecules $\left(E_{S}>1.1 \mathrm{eV}\right)$ can in general be ignored as they do not appreciably desorb from surfaces at room temperature, whereas weakly bound species $\left(E_{S}<0.7 \mathrm{eV}\right)$ can be pumped away quickly. Molecules in the middle of this range are difficult to pump out in a practical time and so high temperature baking is required
[239]. We shall refer to this as the 'Outgassing Energy Range (OER)'.

Outgassing of reactive species from the bulk is more complex than simple diffusion. Gases, such as hydrogen, diffuse ionically and can form bonds with the bulk material or impurities in a process known as trapping. Ions may also recombine within the solid and become trapped in lattice defects, and any ion reaching the surface needs to recombine in order to desorb. At low surface coverage this latter, second order, step can be the limiting rate. Many of the transport processes are activated and so only occur at elevated temperatures. This can result in unreliable predictions when extrapolating high temperature data down to room temperature. The effects are further complicated by surface oxides or nitrides which, in general, act to reduce outgassing rates by providing a barrier layer [240-242]. Noble gases, on the other hand, only travel diffusively through the bulk and easily desorb from surfaces at all temperatures due to their weak interaction. All of the effects outlined above act to reduce the outgassing rate compared to a simple diffusion model and therefore one can assume bulk diffusion as the most significant factor and use the rate calculated by Lewin [239] for a 'slab' geometry:

$$
\frac{Q_{O G}}{A}=\frac{8 x_{0} D}{d} \sum_{m=0}^{\infty} \exp \left[-D t\left(\frac{\pi(2 m+1)}{d}\right)^{2}\right]
$$

Where $D$ is diffusion rate, $A$ is the surface area, $d$ is the thickness and $x_{0}$ is the initial concentration of the gas in the bulk. For a non-disassociative gas $x_{0}=S P$, where $S$ is the solubility and $P$ is the partial pressure of the gas. For a disassociative gas the concentration is proportional to $P^{0.5}$ instead, and the solubility units are adjusted accordingly. By using values for diffusion found experimentally, effects such as trapping are automatically included into the model. As highlighted by Chuntonov et

al [169] the increase of outgassing during the high temperature bonding process can cause the NEG film to become saturated and limit the lowest obtainable vacuum and lifetime of the device. To calculate actual lifetime including the effect of bonding we can consider the reduction of getter capacity by the number of molecules released during bonding, and using Equation 8 to find:

$$
\tau_{L}=\frac{T}{Q}\left(C_{G} R-\frac{\tau_{B}}{T_{B}} \sum_{\text {gases }} Q_{B}\right)
$$

where $T$ is room (or operating) temperature, $T_{B}$ is the bonding temperature, $\tau_{B}$ is the bonding time (seconds), $Q$ is the outgassing rate at $T$, and $Q_{B}$ is the outgassing rate at $T_{B}$. We have assumed the temperatures are changed instantaneously and the bonding period is short enough not to affect the operating outgassing rate. The same formula can be used to predict the increased 
outgassing due to reactivation of the NEG during the MicroMOT lifetime. The effect of the bonding can be neglected if:

$$
\eta=\frac{\tau_{B} Q_{B}}{R C_{G} T_{B}}<<1
$$

Assuming an NEG with a hydrogen capacity of $10^{-7}$ moles $\left(x_{H}=0.01\right)$, a bonding time of $\tau_{B}=3600 \mathrm{~s}$ at a temperature of $T_{B}=400^{\circ} \mathrm{C}$, and we wish to keep $\eta=0.1$, the bonding outgassing rate must be $Q_{B}<$ $10^{-7} \mathrm{mbar} \mathrm{s}^{-1} \mathrm{~cm}^{-2}$. If this is the result of bulk diffusion which scales as

$$
Q=\frac{D(T)}{D\left(T_{B}\right)} Q_{B}=Q_{B} \exp \left(\frac{-E_{D}\left(T-T_{B}\right)}{k_{B} T_{B} T}\right)
$$

and we use a diffusion energy in the middle of the OER of $E_{D}=0.9 \mathrm{eV}$, then one must reduce room temperature hydrogen outgassing rate to $Q_{B}<10^{-16}$ mbar $1 \mathrm{~s}^{-1} \mathrm{~cm}^{-2}$. Equation 15 also applies for surface desorption (for which $E_{D}=E_{S}$ ). Gases such as carbon monoxide, for which the NEG has only a single monolayer capacity, require outgassing rates over a hundred times lower compared to hydrogen without continuous reactivation. These are extremely low outgassing rates and are the main hurdle in obtaining very low vacua in microelectronic devices. In the following subsection we explore the outgassing rates of the main gases found at $\mathrm{UHV}-\mathrm{H}_{2}, \mathrm{CO}$, as well as noble gases - from the materials considered for the MicroMOT and we have tabulated measured and theoretical values for outgassing rates in Table VII.

\section{Hydrogen}

Hydrogen can be a major issue in semiconductor devices and so extensive work has been carried out to understand its interaction with silicon [262-266]. The most common and earliest citation in the literature is to the work done by van Wieringen and Warmoltz [193] (which we shall refer to $\mathrm{vWW}$ ). Their diffusivity and solubility data was taken at very high temperatures $\left(967-1207^{\circ} \mathrm{C}\right)$ and these values are shown in Table V. Extrapolation down to room temperature is fraught with possible errors as hydrogen readily dissociates upon diffusing into the bulk, interacting with the silicon lattice and impurities in various forms. Figure 8 shows the results of subsequent diffusion studies and one can see the large variation in measurements in the literature. Advances in understanding have shown that hydrogen migration through bulk silicon predominantly occurs in atomic form at room temperature with an activation barrier of $\sim 0.5 \mathrm{eV}$, but can recombine into a dimer which is then trapped by a barrier of $0.8-1.2 \mathrm{eV}$. Exact values are difficult to predict and depend on various doping and impurity levels, growth methods, defects and lattice orientations, and even quantum effects [267]. The vWW diffusivity results are consistently higher than every subsequent measurement and can be taken as the upper limit in our analysis [268].

Whichever diffusivity one uses, the vWW solubility results extrapolate to $10^{-10}$ molecules per cubic centimetre at room temperature under 1 bar of $\mathrm{H}_{2}$ and so there should be no hydrogen whatsoever within the bulk. Other measurements have found agreement with a very low value, with the highest at only a few hundred hydrogen atoms per cubic centimetre at room temperature [206, 269, 270]. Using any one of these solubilities does not alter the permeation values in Table $\mathrm{V}$ by a more than a factor of two or three.

Standard semiconductor processing, such as mechanical polishing, HF etching, plasma treatments etc, can result in far higher levels of hydrogen close to the surface [262]. Several studies have found values as high as $10^{18}$ molecules per cubic centimetre and drops significantly after a depth of one micron[271]. This concentration will lead to outgassing rates of $10^{-7} \mathrm{mbar} \mathrm{s}^{-1} \mathrm{~cm}^{-2}$, using Equation 12 and the $\mathrm{vWW}$ diffusivity scaling law, but can be completely degassed to below $10^{-30}$ mbar $1 \mathrm{~s}^{-1} \mathrm{~cm}^{-2}$ within an hour under vacuum as shown in Figure 9, limited by surface recombination. Moreover, surface oxides and nitrides act as efficient permeation barriers [240-242]. Hydrogen bound on the silicon surface has very high desorption energy [272, 273] (above $1.8 \mathrm{eV}$ ) such that the desorption rate from one monolayer coverage would be below $10^{-22} \mathrm{mbar} \mathrm{s}^{-1} \mathrm{~cm}^{-2}$ using Equation 11. Thermal desorption studies [273] show that most hydrogen complexes can be desorbed from silicon by annealing at $600^{\circ} \mathrm{C}$.

Hickmott [274] studied the interaction between hydrogen and glass and found that the hot filament of the ionization gauge had a detrimental effect on determining the residual gas content at UHV. He noted that hydrogen was desorbed at the two distinct activation energies of $0.29 \mathrm{eV}$ and $1.08 \mathrm{eV}$. The former is so low that it will desorb completely at room temperature under vacuum, whereas the latter requires baking above $400^{\circ} \mathrm{C}$. Spectroscopic studies by Hickmott showed that after a high temperature bake the main residual gases were water and carbon monoxide. Todd [275] measured the residual water composition in a variety of glasses and found negligible outgassing $\left(\sim 10^{-23} \mathrm{mbarl}^{-1} \mathrm{~cm}^{-2}\right)$ in AS glass after high temperature baking. This low outgassing rate is due to the strong $\mathrm{Si}-\mathrm{H}$ and $\mathrm{Si}-\mathrm{OH}$ bonds. Using the values from Table $\mathrm{V}$ and Equation 12 to calculate the lowest hydrogen outgassing rate from AS glass, as shown in Table VII, we find remarkable agreement between the theoretical value

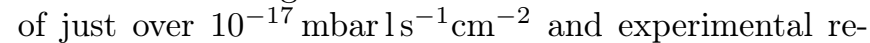

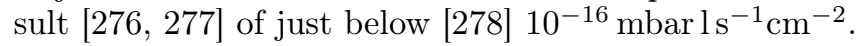




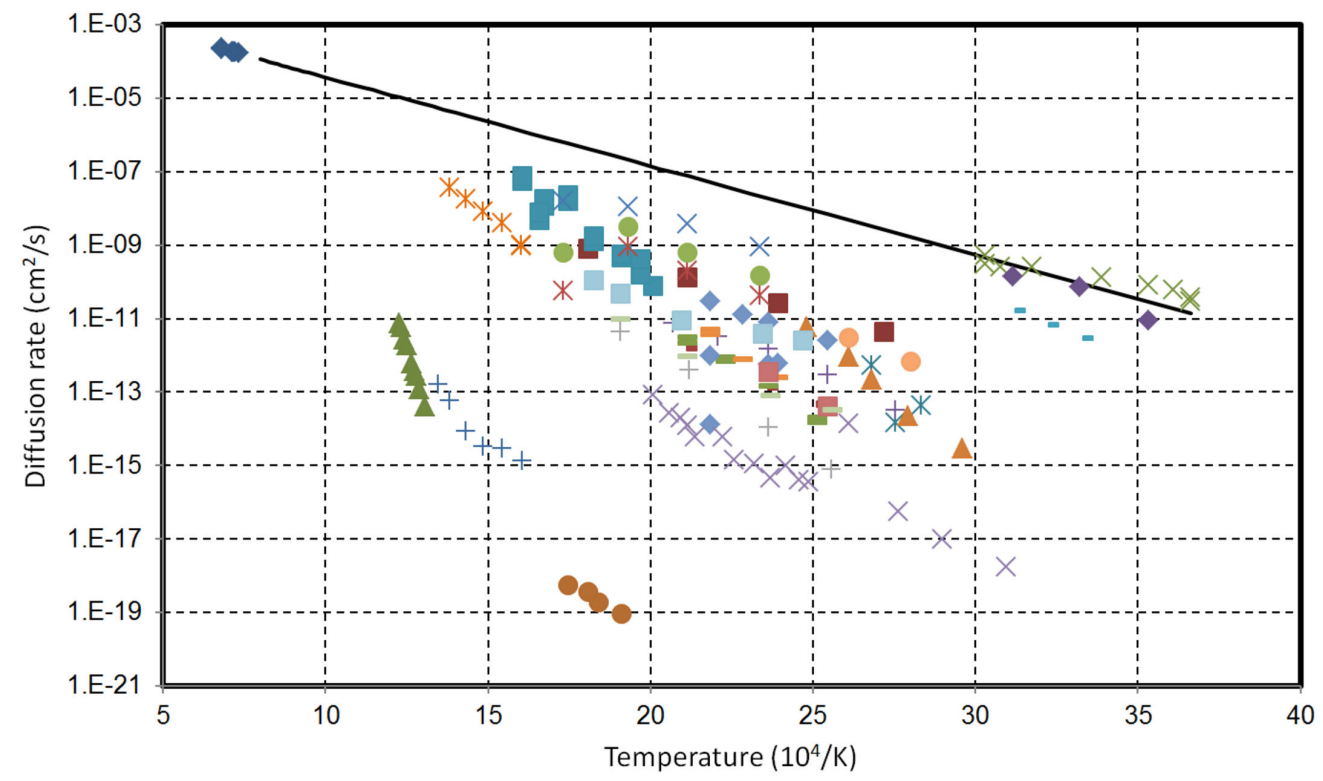

- Van Wieningen et al (1956) CZ

- Koveshnikov et al (1990)

- Kamiura et al (1991)

$\times$ Seager et al (1998)

* Schmalz et al (1989) CZ p-type

- Vergnat et al (1991) Poly

+ Tang et al (1990) Poly

- Johnson et al (1991) CZ n-type

- Johnson et al (1991) FZ n-type

- Seager et al (1990)

- Newman et al (1991) CZ

- Majumdar et al (1997)

$\times$ Sopori et al (1992) Poly

* Sopori et al (1992) CZ

- Sopori et al (1992) FZ

+ Herrero et al (1990) CZ p-type

- Johnson et al (1992)

- Rizk et al (1991) HO p-type

- Rizk et al (1991) H+ p-type

- Rizk et al (1991) H0 n-type

$\triangle$ Rizk et al (1991) H- n-type

$\times$ Fabian et al (1998)

* Huang et al (2004)

- Hansen et al (1984)

+ Capizzi et al (1987) HO

- Capizzi et al (1987) $\mathrm{H}+$

Mogro-Campero et al (1985)

FIG. 8. The large scatter in data for diffusion of hydrogen through silicon [193, 243-261]. We have only plotted data for hydrogen diffusion (no isotopes) and have indicated the type of silicon where known. The solid black line is the commonly quoted vWW diffusion rate.

\section{Noble gases}

Noble gases cannot be removed once the MicroMOTs are sealed and so must be completely degassed from all components before bonding. As discussed in Section V B negligible levels of noble gases, specifically helium, should be found in silicon unless additional data corroborates a recent study [207]. Noble gases in glasses are expected to reach concentrations of $10 \mathrm{ppb}$ when exposed to atmosphere, which will outgas from the bulk diffusively. Figure 10 shows the results of degassing AS glass using Equation 12, and we see that a thin slab can be completely degassed easily. This would scale proportionally to the area when wafer level degassing is required.

The last materials in our chips are metals such as gold for the atom chip and those for the NEG. Noble gases do not permeate most metals and so one should be able to ignore these materials, however the method of their deposition is important: Sputtered thin films have been found to incorporate large quantities of argon, as this process gas is used to remove the metal atoms from the sputter target. The argon is then buried in the growing thin film and permeates to the surface along dislocations and pores, as well as via self-diffusion. Where possible films should be deposited by vacuum arc deposition [156] or e-beam evaporation which do not require additional gases. In situations where sputtering is unavoidable several modifications can be made to reduce contamination by this gas source including $[165,166]$ : increasing the sputter gas pressure [279], increasing the substrate temperature, reducing the deposition rate, post annealing, and finally changing to a heavier gas. Using this last modification by replacing argon with krypton has been shown to reduce the gas incorporation by a factor of $10^{3}$ or even lower, but moving to xenon shows little improvement [166]. Measurements of Ti-Zr-V NEG films have detected krypton outgassing rates [280] at the sensitivity limit of the detector - down to $10^{-19} \mathrm{mbar} \mathrm{l} \mathrm{s}^{-1} \mathrm{~cm}^{-2}$ after several activations - which is still too high for our device, but this could be further reduced with higher temperature anneals.

\section{Carbon monoxide}

Carbon monoxide is the second most significant gas at UHV. Due to its relatively large size (compared to helium or hydrogen) diffusion through the bulk will be negligible and so carbon monoxide is largely a surface outgassing species [281]. Studies looking at the residual gases in glass have shown that the carbon monoxide concentrations vary widely [282] and it is difficult to obtain repeatable results. In addition, this also depends on glass type, processing history, and the effects of ionization cathodes. We do not know the desorption energy for carbon monoxide on glass, but due to the latter's low reactivity we expect carbon monoxide to only be physisorbed and so quickly degassed. Similar conclusions have been noted in photodesorption measurements [283]. One study looking at the effect of breaking glass substrates in vacuum found that the carbon monoxide level remained constant, but carbon dioxide quickly decreased [284]. This was attributed to the reaction with residual hydrogen forming methane, which was seen to increase. 


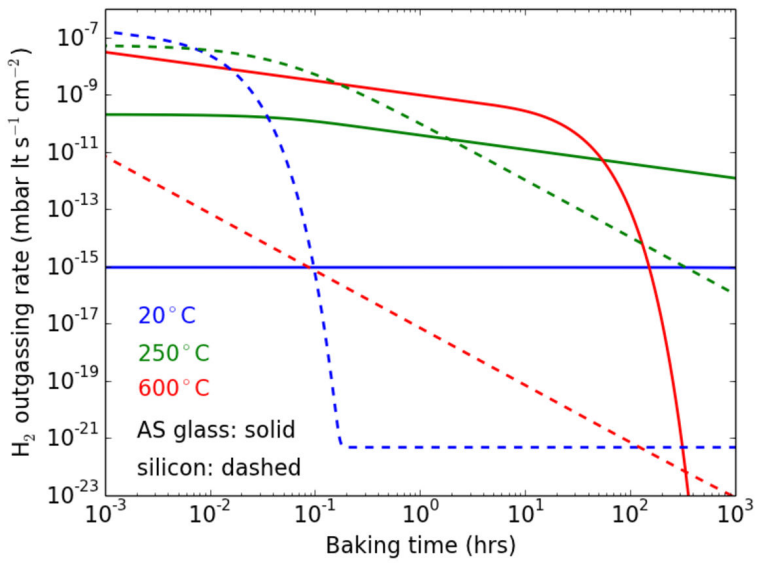

FIG. 9. The theoretical outgassing rate of hydrogen for $1 \mathrm{~mm}$ thick silicon (dashed line) and AS glass (solid line) for three baking temperatures. We have assumed AS glass is diffusion limited and calculated the rate using Equation 12 and values from Table $\mathrm{V}$ after exposure to 1 bar hydrogen (i.e. immersion into water). We have calculated the rate for silicon using a summation of diffusion (Equation 12) from the bulk as well as considering the higher concentration at the surface (see text), and also recombination-limited surface desorption using Equation $11(k=2)$ with values from Gupta et al [273]. The room temperature silicon outgassing shows an initially high rate due to diffusion of the high concentration near the surface and is eventually limited by surface desorption of the dihydride surface species (as are the higher temperature bakes).

A careful study [285] avoiding the effects of gauges measured carbon monoxide outgassing rates from stainless steel less than $6 \times 10^{-17} \mathrm{mbarls}^{-1} \mathrm{~cm}^{-2}$, three orders of magnitude less than hydrogen.

Thermal desorption studies of carbon monoxide released from silicon surfaces are few, but show that the thermal desorption energy is below $0.5 \mathrm{eV}[286]$ and so should be degassed easily. Photodesorption and plasma cleaning have also been shown to efficiently remove carbon and oxygen contamination from silicon surfaces.

\section{Other gases}

Methane is commonly found in UHV environments is not pumped by Ti-Zr-V getters at room temperature. It is thought to be produced from reactions on the high temperature electrodes of ionization gauges, so should not be an issue in our gauge-less MicroMOTs. It may also be formed from reactions between carbon monoxide and hydrogen during their diffusion on NEG or glass surfaces [284], as mentioned before. A desorption rate of $10^{-14}$ mbar lt s $\mathrm{cm}^{-1}$ of methane was measured by Setina et al [287] from Ti-Zr-V-Fe getter strips which would be a serious vacuum risk in the MicroMOTs, however one would expect the methane production rate to be related to the partial pressures of $\mathrm{H}_{2}$ and $\mathrm{CO}$ which was

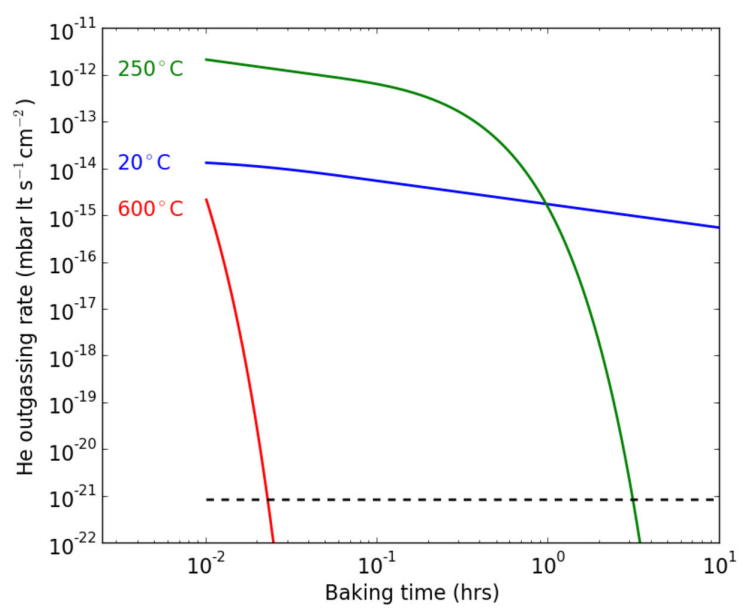

FIG. 10. The He outgassing rate from $1 \mathrm{~mm}$ thick aluminosilicate glass slab at various temperatures after storage in atmosphere (5 ppm He content), calculated with Equation 12. The black dashed line indicates the target outgassing rate.

not seen in other studies [288] below $10^{-8}$ mbar. Therefore, methane should be completely evacuated before the MicroMOT is sealed, otherwise one must use reactive getters, or briefly elevate the NEG temperature to achieve some pumping. Other organic species have been found in encapsulated MEMs-type devices which are likely due to insufficent cleaning or residual gases prior to sealing [132, 289].

Should anodic bonding be used to seal the chips, oxygen will be released along the inner bonding edges where the voltage is high and no silicon exists to bond with the non-bridging oxygen atoms [181, 221, 290]. Predicting the amount of oxygen released is unreliable due to the lack of data and the effects of bonding parameters, chip dimensions, and increased outgassing at raised temperatures. However, by analyzing the few studies on this subject $[221,290,291]$ we estimate $10^{13}$ to $10^{14}$ molecules per millimetre inner bonding circumference. For our MicroMOT design this can lead to a monolayer coverage of oxygen on the NEGs and result in saturation. It should be noted that oxygen penetrates the NEG surface resulting in a capacity of about five monolayers [157]. Therefore it is important to maintain the chip at high temperatures after bonding to absorb the oxidized NEG layers into the bulk. Once rubidium is released into the chip it will quickly oxidize with any remaining oxygen forming $\mathrm{Rb}_{2} \mathrm{O}$, which also reacts exothermically with water and hydrogen forming stable hydroxides and hydrides which do not contaminate vacuum.

\section{E. Vacuum discussion}

We have identified all the main sources of residual gases which could threaten our sealed UHV environment. We 
TABLE VII. Lowest and typical room temperature outgassing rates for $2 \mathrm{~mm}$ thick materials. The theoretical values (Th.) have been estimated using Equation 12 and Figure 9 with a $10 \mathrm{hr} 250^{\circ} \mathrm{C}$ vacuum bake for 'typical outgassing' and an additional $1 \mathrm{hr} 600^{\circ} \mathrm{C}$ vacuum bake for 'lowest outgassing'.

\begin{tabular}{lcc}
\hline $\begin{array}{l}\text { Outgassing rate } \\
\left(\mathrm{mbar} \mathrm{s} \mathrm{s}^{-1} \mathrm{~cm}^{-2}\right)\end{array}$ & Lowest & Typical \\
\hline Silicon $^{\mathrm{a}}$ & $10^{-30}$ (Th.) & $10^{-24}$ (Th.) \\
Aluminosilicate & $10^{-17}$ & $10^{-16}$ (Th. $\left.^{\mathrm{b}}\right)$ \\
Pyrex & $10^{-14}$ & $10^{-10}$ \\
Stainless steel & $10^{-15 \mathrm{c}}$ & $10^{-12}$ \\
\hline
\end{tabular}

a Theoretical values taken from Figure 9.

b Using values from Table $\mathrm{V}$

c Thinner materials have lower outgassing rates and Nemanič et al [298] have demonstrated $10^{-17}$ with $150 \mu \mathrm{m}$ foil.

have seen that helium permeation through glass can be reduced to a negligible level with the use of aluminosilicates and could further be improved with optical coatings such as graphene [191]. Leaking through bonds must be several orders of magnitude lower than has been measured, but can be sufficiently improved by incorporating a 'moat' within the bonding seam. We also note that leakage can be further reduced by coating the inner edges of bonding seams with NEG films and by applying a barrier coating on the outer edges of the device. Several bonding techniques are available and we highlight eutectic and direct bonding as the most suitable methods due to their low outgassing and high hermeticity, with anodic bonding as a suitable and simpler alternative if the oxygen released during bonding can be pumped away. Lowering the temperatures of these bonding techniques should be investigated as they can reduce the outgassing limitations by two or three orders of magnitude [219, 223, 224, 228, 292-297].

The greatest hurdle we are left with is to reduce outgassing. This can be tackled in two ways: 1) improve the pumping rate and capacity of the getter films, and 2) reduce the outgassing rate by extensive degassing procedures and careful choice of materials.

It has been shown that the pumping rate of NEGs is difficult to improve even with reactive lithium getters, however the latter retains a constant pumping rate irrespective of its history. NEGs are more straightforward to deposit, can be used to coat surfaces to reduce outgassing and are stable in air. Reactive getters need to be deposited under vacuum and could result in unwanted coating on components in the chamber. However, they have far higher capacities and can pump gases such as methane, which NEGs cannot. Therefore we see a combination of NEGs and reactive getters as a good compromise with the former activated during bonding and the latter activated after bonding.

For the second method to tackle outgassing we have seen in Table VII that at room temperature the materials we have chosen for the device are more than adequate once degassed to achieve the room temperature (hydro- gen) outgassing rate of $10^{-12} \mathrm{mbarl} \mathrm{s}^{-1} \mathrm{~cm}^{-2}$ (Equation 8). When we consider the outgassing with a bonding process at around $400^{\circ} \mathrm{C}$ during which outgassing can increase by eight orders of magnitude and put stricter room temperature rates of less than $10^{-16} \mathrm{mbarls}^{-1} \mathrm{~cm}^{-2}$ (Equation 14) for bulk gettered gases such as hydrogen, $10^{-18} \mathrm{mbarl} \mathrm{s}^{-1} \mathrm{~cm}^{-2}$ for surface gettered gases such as carbon monoxide, and less than $10^{-21} \mathrm{mbar} \mathrm{l} \mathrm{s}^{-1} \mathrm{~cm}^{-2}$ for non-gettered noble gases. We can see in Table VII that silicon outgassing is likely to be negligible compared to AS glass whose rate matches our target. When calculating this latter value we assumed a diffusion energy of $0.9 \mathrm{eV}$. We can now be confident that AS glass will be the major source of hydrogen so if we use a more realistic value of $0.79 \mathrm{eV}$ (Table $\mathrm{V}$ ) we lower our target to $10^{-15} \mathrm{mbarl} \mathrm{s}^{-1} \mathrm{~cm}^{-2}$, which is certainly achievable. Carbon monoxide outgassing is difficult to predict but we expect it to be far lower than hydrogen, as found in stainless steel [285]. We have seen that noble gases may be sufficiently removed from the chamber material with realistic baking parameters and by using the separated wafer fabrication method shown in Figure 4, one can ensure the optimum baking regime for each material.

\section{PROTOTYPE MICROMOT}

We are now at the stage where a prototype MicroMOT can be designed to provide realistic values from which we can estimate the vacuum performance and lifetime. We are currently in the process of fabricating these devices based on the following design which we will characterize and present detailed processes at a later date. We have consciously avoided designing the MicroMOT around a single application or manipulation technique, e.g. free-falling atom interferometers, or BECs on atom chips. This is because the MicroMOT will likely need to be adapted for the specific task, so we have chosen a simple generic design to demonstrate what is possible.

As discussed in Section II we assume a G-MOT type geometry using a $10 \mathrm{~mm}$ diameter grating structure and a cavity volume of $15 \times 15 \times 3 \mathrm{~mm}^{3}$ to avoid light scattering off sidewalls. Around $90 \%$ of the beam overlap volume is within $2.5 \mathrm{~mm}$ of the grating surface, and so this is a reasonable choice of height and is also feasible to fabricate from silicon using deep reactive ion etching, wet etching, machining, or powder blasting [299]. The chip is formed of four chambers: a large science chamber for cooling and manipulating the atoms, an atom source chamber in which to hold the alkali dispensers, an alkali getter/LIAD/peltier chamber, and a reactive getter chamber. The source chamber is connected to the science chamber by a very thin channel to restrict the vapour flow. The top $2 \mathrm{~mm}$ thick capping wafer is antireflection coated and is anodically or direct bonded to the silicon 'cavity wafer'. The thickness of the glass layer is determined by several factors including the permeation rates, bondability, structural integrity and price. This 


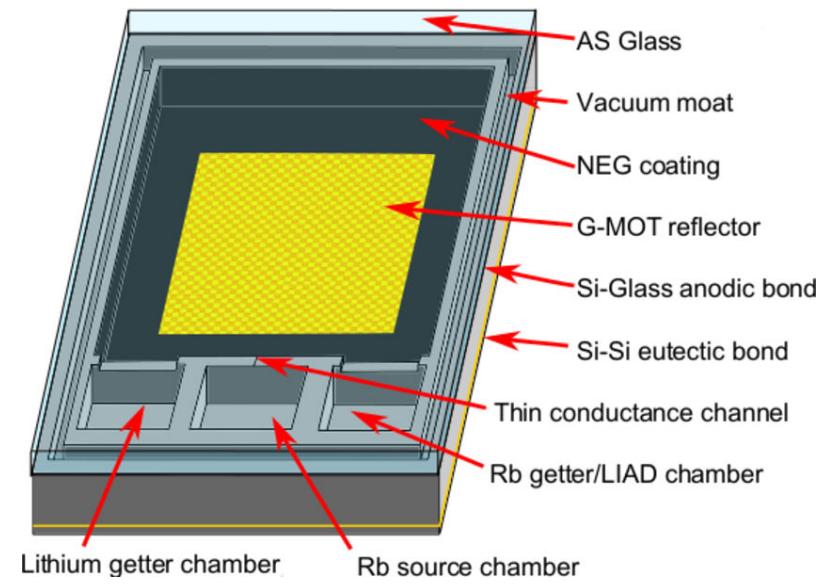

FIG. 11. Prototype design of a miniaturized magneto-optical trap incorporating all the elements discussed in the text. Dimensions are $20 \times 24 \times 5 \mathrm{~mm}^{3}$.

wafer incorporates a moat within the bonding region on both sides to reduce argon leakage. The reflector layer is coated by a thin alumina layer to prevent alkali corrosion of the gold and is eutectically bonded to the cavity wafer. Future designs will include atom chip wires and electrical feedthroughs forming this chamber wall. We have not shown the quadrupole magnetic field coils as these are trivial to implement and may simply be bonded, or deposited, onto the top and bottom external surfaces, or could be approximated with a double loop on single surface [300].

Following extensive cleaning and baking procedures, the glass and silicon wafers are first bonded in vacuum so that the oxygen released during anodic bonding can be removed without saturating the NEG. An NEG thin film is sputtered on the internal sidewalls and onto some of the bottom layer (with the reflector masked off) to provide the largest NEG area and reduce outgassing. The remaining reflector layer is then eutectically bonded under UHV after high temperature baking to desorb hydrogen, carbon monoxide and noble gases.

Table VIII provides a detailed summary of dimensions and an estimation on the vacuum properties of the device using values calculated in this study. We can see that the lifetime of the device is determined by the argon leakage, but is nearly an order of magnitude greater than our target. The extremely low outgassing rate of AS glass results in negligible outgassing after bonding such that the base pressure is in the XHV regime for an essentially unlimited lifetime. Practically, the lifetime and base pressure will be determined by the atom source and the residual noble gas partial pressure in the bonding chamber which we typically achieve levels below $10^{-11}$ mbar.

\section{DISCUSSION AND CONCLUSIONS}

We have shown that it is feasible to maintain UHV, and even XHV, environments for extended periods inside sealed chips using materials and methods borrowed from the semiconductor industry. However, it is necessary to highlight the assumptions we have made if these type of devices are to become a realistic technology. A great emphasis has been made on degassing the materials at temperatures up to $600^{\circ} \mathrm{C}$ to ensure a sufficiently low outgassing rate once sealed. For the bulk materials discussed this is certainly possible, but more sophisticated devices are likely to have additional components, such as micro-Peltier coolers, detectors, field emission tips (for active pumping or ionization), or even light sources (such as UV LEDs for LIAD), which can be sensitive to extreme temperatures. Moreover, thin films, such as gold on silicon, can diffuse at moderate temperatures if additional barrier layers are not used [218]. In these situations one must use lower temperature degassing, such as UV desorption or plasma cleaning, and also develop lower temperature bonding methods [219, 223, 224, 228, 292-297].

The very low leak rates we have predicted are possible by a combination of hermetic bonding and additional moat cavities. We have assumed that the materials to be joined are reliably homogeneous and intact (e.g. perfect crystallinity in the case of silicon) but in reality fabrication processes may lead to defects which can result in additional leakage routes such as microcracks, crystal plane dislocations, surface defects, or thin oxide films. Surface barrier films, NEG coatings and stress-relief annealing can reduce these effects but these possible sources of leakage are still worth bearing in mind.

We have used the large amount of data on hydrogen diffusion in silicon to predict that it has a very low outgassing rate. We find that this rate is consistently low whichever values we use from the literature, especially after a high temperature bake for several hours, and so we are confident in the estimate. However, as mentioned in Section VD, there is no absolutely reliable method to predict the outgassing rate from real materials and the simple diffusion-limited model is only useful to an order of magnitude at best, especially when considering the migration of reactive species such as hydrogen. This can be seen with studies looking at the outgassing of stainless steel, where the diffusion limited model produces reasonable estimate for low temperature bakes (below $300^{\circ} \mathrm{C}$ ), but generally fails to predict the effect of very high temperatures. This is usually attributed to the effect of surface oxides which are more stable during low temperature baking and act as diffusion barriers [301]. Therefore we expect silicon to have a very low outgassing rate but probably higher than the value stated in Table VII. Experimental studies focusing specifically on outgassing are required. We have used measured values for AS glass outgassing, but glasses are notorious for producing variable results [302] so it is necessary to perform additional outgassing studies on the specific glass one uses to ensure 
TABLE VIII. Expected vacuum properties of the prototype MicroMOT in atmosphere at $20^{\circ} \mathrm{C}$.

\begin{tabular}{|c|c|c|c|}
\hline Internal volume $\left(\mathrm{cm}^{3}\right)$ & 0.65 & including subchambers & \\
\hline Surface area $\left(\mathrm{cm}^{2}\right)$ & 7.8 & 2.6 glass & \\
\hline $\mathrm{NEG}^{\mathrm{a}}$ area $\left(\mathrm{cm}^{2}\right)$ & 3.3 & all sides and some of the reflector surface & \\
\hline Glass thickness $(\mathrm{cm})$ & 0.2 & & \\
\hline Bonder base pressure (mbar) & $10^{-9}$ & equal $\mathrm{H}_{2}$ and $\mathrm{CO}$, negligible noble gases & \\
\hline Bonding parameters & $1 \mathrm{hr}$ at $400^{\circ} \mathrm{C}$ & typical eutectic bond & \\
\hline Gas source $\left(\right.$ mbarls $\left.\mathrm{s}^{-1}\right)$ & Surface pumped (CO) & Bulk pumped $\left(\mathrm{H}_{2}\right)$ & Noble gas \\
\hline Leakage $^{\mathrm{c}}$ & $1.9 \times 10^{-23}$ & $3.8 \times 10^{-24}$ & $2.3 \times 10^{-22}$ \\
\hline Outgassing $^{\mathrm{d}}$ & $4.5 \times 10^{-17}$ & $2.6 \times 10^{-16}$ & $<10^{-24}$ \\
\hline Base pressure $^{\mathrm{e}}$ (mbar) & $10^{-18}$ & $10^{-15}$ & - \\
\hline Lifetime (days to reach $1.5 \times 10^{-10} \mathrm{mbar}$ ) & $10^{6}$ & $10^{9}$ & 3200 \\
\hline \multicolumn{4}{|c|}{$\begin{array}{l}\text { a Surface capacity of } 5 \times 10^{14} \text { molecules } \mathrm{cm}^{-2} \text { and bulk capacity of } 7.5 \times 10^{16} \text { molecules } \mathrm{cm}^{-2}\left(1 \mu \mathrm{m} \text { film with } x_{H}=0.01\right) \text {. } \\
\text { b The permeation rates are calculated using the breakthrough time from Equation } 4 \text { substituted into Equation } 5 \text {. } \\
\text { c Bond leak rate (air) of } 10^{-15} \text { mbar } \mathrm{s}^{-1} \text { for } 2 \text { bonding seams (top wafer and bottom wafer). We have assumed the carbon monoxide } \\
\text { leak is from atmospheric carbon dioxide. } \\
\text { d As the carbon monoxide outgassing rate is unknown we have assumed a value which is ten times less than hydrogen as discussed in the } \\
\text { text as a worst case scenario. }\end{array}$} \\
\hline
\end{tabular}

suitability. For the sake of bevity we have limited our discussions to silicon and glasses but there are likely to be many other suitable materials, most noteably ceramics.

In conclusion, the aim of this study was to prove that Magneto-Optical Traps can be miniaturised and integrated into devices capable of leaving the laboratory. We have shown that recent advances in microfabrication techniques and materials can lead to sealed chambers with microlitre volumes that maintain UHV for at least 1000 days using only passive pumping elements. The MOT geometry can be miniaturized to use a single laser beam, patterned reflectors, planar optics, and atom source. Controlled by a number of methods including LIAD, integrated cold fingers, conductance channels and several pumping mechanisms. The main issues to maintain sealed UHV environments are the need for ex- tremely low leakage bonds, low outgassing materials, and also negligible noble gas outgassing from chamber walls and sputtered films. We hope that this work motivates the development of ultracold quantum technology which has a vast number of practical applications and promises to be a fruitful technology in a number of fields.

\section{ACKNOWLEDGMENTS}

We would like to acknowledge Tim Freegarde, David Smith, James Gates, Peter Smith, Erling Riis, Aidan Arnold, Joe Cotter, Wolfgang Reinert, and Kai Bongs, for their helpful and insightful advice and ideas in the development of this study. This work was supported by the Royal Academy of Engineering, pdfRC, the Royal Society, and DSTL.
[1] B. Barrett, P.-A. Gominet, E. Cantin, L. AntoniMicollier, A. Bertoldi, B. Battelier, P. Bouyer, J. Lautier, and A. Landragin, arXiv preprint arXiv:1311.7033 (2013).

[2] J. Kitching, S. Knappe, and E. A. Donley, Sensors Journal, IEEE 11, 1749 (2011).

[3] N. Hinkley, J. A. Sherman, N. B. Phillips, M. Schioppo, N. D. Lemke, K. Beloy, M. Pizzocaro, C. W. Oates, and A. D. Ludlow, Science 341, 1215 (2013).

[4] S. M. Dickerson, J. M. Hogan, A. Sugarbaker, D. Johnson, and M. A. Kasevich, arXiv preprint arXiv:1305.1700 (2013).

[5] B. Canuel, F. Leduc, D. Holleville, A. Gauguet, J. Fils, A. Virdis, A. Clairon, N. Dimarcq, C. J. Bordé, A. Landragin, et al., Physical review letters 97, 010402 (2006).
[6] C. J. Bordé, Metrologia 39, 435 (2002).

[7] F. Yver-Leduc, P. Cheinet, J. Fils, A. Clairon, N. Dimarcq, D. Holleville, P. Bouyer, and A. Landragin, Journal of Optics B: Quantum and Semiclassical Optics 5, S136 (2003).

[8] M. Edwards, Nature Physics 9, 68 (2013).

[9] D. Budker and M. Romalis, Nature Physics 3, 227 (2007).

[10] N. Behbood, F. Martin Ciurana, G. Colangelo, M. Napolitano, M. W. Mitchell, and R. J. Sewell, Applied Physics Letters 102, 173504 (2013).

[11] S. Wildermuth, S. Hofferberth, I. Lesanovsky, S. Groth, P. Kruger, J. Schmiedmayer, and I. Bar-Joseph, Applied physics letters 88, 264103 (2006).

[12] D. Oblak, P. G. Petrov, C. L. G. Alzar, W. Tittel, A. K. 
Vershovski, J. K. Mikkelsen, J. L. Sørensen, and E. S. Polzik, Physical Review A 71, 043807 (2005).

[13] M. Napolitano, M. Koschorreck, B. Dubost, N. Behbood, R. Sewell, and M. W. Mitchell, Nature 471, 486 (2011).

[14] S. Boixo, A. Datta, M. J. Davis, S. T. Flammia, A. Shaji, and C. M. Caves, Physical review letters 101, 040403 (2008).

[15] L. Pezzé and A. Smerzi, Physical review letters 102, 100401 (2009).

[16] J. Appel, P. J. Windpassinger, D. Oblak, U. B. Hoff, N. Kjærgaard, and E. S. Polzik, Proceedings of the National Academy of Sciences 106, 10960 (2009).

[17] R. Sewell, M. Koschorreck, M. Napolitano, B. Dubost, N. Behbood, and M. Mitchell, Physical review letters 109, 253605 (2012).

[18] C. Monroe, Nature 416, 238 (2002).

[19] A. Kozhekin, K. Mølmer, and E. Polzik, Physical Review A 62, 033809 (2000).

[20] L.-M. Duan, M. Lukin, J. I. Cirac, and P. Zoller, Nature 414, 413 (2001).

[21] S. Diehl, A. Micheli, A. Kantian, B. Kraus, H. Büchler, and P. Zoller, Nature Physics 4, 878 (2008).

[22] H. Marion, F. P. Dos Santos, M. Abgrall, S. Zhang, Y. Sortais, S. Bize, I. Maksimovic, D. Calonico, J. Grünert, C. Mandache, et al., Physical review letters 90, 150801 (2003).

[23] S. Dimopoulos, P. W. Graham, J. M. Hogan, and M. A. Kasevich, Physical review letters 98, 111102 (2007).

[24] C. J. Bordé, Philosophical Transactions of the Royal Society A: Mathematical, Physical and Engineering Sciences 363, 2177 (2005).

[25] J. P. Dowling and G. J. Milburn, Philosophical Transactions of the Royal Society of London. Series A: Mathematical, Physical and Engineering Sciences 361, 1655 (2003).

[26] J. Pritchard and S. Till, "UK Quantum Technology Landscape 2014,".

[27] T. Könemann, W. Brinkmann, E. Göklü, C. Lämmerzahl, H. Dittus, T. Van Zoest, E. Rasel, W. Ertmer, W. Lewoczko-Adamczyk, M. Schiemangk, et al., Applied Physics B 89, 431 (2007).

[28] R. Geiger, V. Ménoret, G. Stern, N. Zahzam, P. Cheinet, B. Battelier, A. Villing, F. Moron, M. Lours, Y. Bidel, et al., Nature communications 2, 474 (2011).

[29] T. Van Zoest, T. Müller, T. Wendrich, M. Gilowski, E. Rasel, W. Ertmer, T. Könemann, C. Lämmerzahl, H. Dittus, A. Vogel, et al., International Journal of Modern Physics D 16, 2421 (2007).

[30] S. Groth, P. Kruger, S. Wildermuth, R. Folman, T. Fernholz, J. Schmiedmayer, D. Mahalu, and I. BarJoseph, Applied Physics Letters 85, 2980 (2004).

[31] J. Reichel and V. Vuletic, Atom Chips (John Wiley \& Sons, 2010).

[32] D. Stick, W. Hensinger, S. Olmschenk, M. Madsen, K. Schwab, and C. Monroe, Nature Physics 2, 36 (2005).

[33] M. Brownnutt, G. Wilpers, P. Gill, R. Thompson, and A. Sinclair, New Journal of Physics 8, 232 (2006).

[34] Symmetricom SA.45 chip scale atomic clock.

[35] S. Knappe, V. Gerginov, P. Schwindt, V. Shah, H. Robinson, L. Hollberg, and J. Kitching, Optics Letters 30, 2351 (2005).

[36] M. de Angelis, M. Angonin, Q. Beaufils, C. Becker,
A. Bertoldi, K. Bongs, T. Bourdel, P. Bouyer, V. Boyer, S. Dörscher, et al., Procedia Computer Science 7, 334 (2011).

[37] S. Chu, Reviews of Modern Physics 70, 685 (1998).

[38] K. Salit, J. Sebby-Strabley, K. Nelson, and J. Kriz, in Frequency Control Symposium (FCS), 2012 IEEE International (IEEE, 2012) pp. 1-4.

[39] B. Xu, X. Chen, J. Wang, and M. Zhan, Optics Communications 281, 5819 (2008).

[40] V. Shah, M. Mescher, R. Stoner, V. Vuletic, and R. Lutwak, arXiv preprint arXiv:1111.5890 (2011).

[41] E. A. Salim, J. DeNatale, D. M. Farkas, K. M. Hudek, S. E. McBride, J. Michalchuk, R. Mihailovich, and D. Z. Anderson, Quantum Information Processing 10, 975 (2011).

[42] R. Folman, P. Krüger, D. Cassettari, B. Hessmo, T. Maier, and J. Schmiedmayer, Physical Review Letters 84, 4749 (2000).

[43] S. Wildermuth, P. Krüger, C. Becker, M. Brajdic, S. Haupt, A. Kasper, R. Folman, and J. Schmiedmayer, Physical Review A 69, 030901 (2004).

[44] K. Lee, J. Kim, H. Noh, and W. Jhe, Optics letters 21, 1177 (1996).

[45] J. Arlt, O. Marago, S. Webster, S. Hopkins, and C. Foot, Optics communications 157, 303 (1998).

[46] M. Vangeleyn, P. F. Griffin, E. Riis, and A. S. Arnold, Optics express 17, 13601 (2009).

[47] M. Trupke, F. Ramirez-Martinez, E. Curtis, J. Ashmore, S. Eriksson, E. Hinds, Z. Moktadir, C. Gollasch, M. Kraft, G. Vijaya Prakash, et al., Applied physics letters 88, 071116 (2006).

[48] S. Pollock, J. Cotter, A. Laliotis, and E. Hinds, Optics Express 17, 14109 (2009).

[49] S. Pollock, J. Cotter, A. Laliotis, F. Ramirez-Martinez, and E. Hinds, New Journal of Physics 13, 043029 (2011).

[50] This can be mitigated with background free imaging [303].

[51] C. Nshii, M. Vangeleyn, J. Cotter, P. Griffin, E. Hinds, C. Ironside, P. See, A. Sinclair, E. Riis, and A. Arnold, Nature nanotechnology (2013).

[52] J. Lee, J. Grover, L. Orozco, and S. Rolston, JOSA B 30, 2869 (2013).

[53] E. Riis, J. McGilligan, and A. Arnold, in ICAP 2014 Proceedings (2014) pp. Tues-058.

[54] Q. Bodart, S. Merlet, N. Malossi, F. P. Dos Santos, P. Bouyer, and A. Landragin, Applied Physics Letters 96, 134101 (2010).

[55] M. A. Perez, U. Nguyen, S. Knappe, E. A. Donley, J. Kitching, and A. M. Shkel, Sensors and Actuators A: Physical 154, 295 (2009).

[56] G. W. Hoth, E. A. Donley, and J. Kitching, Optics Letters 38, 661 (2013).

[57] Several studies have shown the scaling laws for various beam overlap volumes, however we highlight that none have considered the effect of unsymmetrical volumes for which reducing one dimension could be beneficial in wafer-level microfabrication by reducing chamber size whilst maintaining a large capture volume.

[58] L. Huet, M. Ammar, E. Morvan, N. Sarazin, J.-P. Pocholle, J. Reichel, C. Guerlin, and S. Schwartz, in International Quantum Electronics Conference (Optical Society of America, 2011) p. I1188.

[59] A. V. Rakholia, H. J. McGuinness, and G. W. Bieder- 
mann, arXiv preprint arXiv:1407.3847 (2014).

[60] T. Kovachy, J. M. Hogan, D. M. Johnson, and M. A. Kasevich, Physical Review A 82, 013638 (2010).

[61] A. Sahm, C. Fiebig, S. Spiesberger, M. Schiemangk, E. Luvsandamdin, K. Paschke, G. Erbert, and G. Trankle, in Electronic Components and Technology Conference (ECTC), 2012 IEEE 62nd (IEEE, 2012) pp. 18521857.

[62] C. Grzeschik, M. Schiemangk, K. Lampmann, M. Krutzik, and A. Peters, in CLEO: Science and Innovations (Optical Society of America, 2013) pp. CM1N-6.

[63] S. Spießberger, M. Schiemangk, A. Sahm, A. Wicht, H. Wenzel, A. Peters, G. Erbert, and G. Tränkle, Optics express 19, 7077 (2011).

[64] K. Numata, M. Alalusi, L. Stolpner, G. Margaritis, J. Camp, and M. Krainak, Optics letters 39, 2101 (2014).

[65] S. Lynch, F. Chen, J. Gates, C. Holmes, S. Staines, S. James, J. Hodgkinson, P. Smith, and R. Tatam, in SPIE OPTO (International Society for Optics and Photonics, 2014) pp. 900209-900209.

[66] K. Numata, J. Camp, M. A. Krainak, and L. Stolpner, Optics express 18, 22781 (2010).

[67] T. Lévèque, L. Antoni-Micollier, B. Faure, and J. Berthon, Applied Physics B , 1 (2013).

[68] G. T. Reed, G. Mashanovich, F. Gardes, and D. Thomson, Nature photonics 4, 518 (2010).

[69] Y. Shoji and T. Mizumoto, Science and Technology of Advanced Materials 15, 014602 (2014).

[70] S. Sheard, T. Suhara, and H. Nishihara, Lightwave Technology, Journal of 11, 1400 (1993).

[71] K. Mehta, A. Eltony, I. Chuang, and R. Ram, in ICAP 2014 Proceedings (2014) pp. Tues-077.

[72] R. Shechter, Y. Amitai, and A. A. Friesem, Applied optics 41, 1236 (2002).

[73] O. Alloschery, R. Mathevet, J. Weiner, and H. Lezec, Optics express 14, 12568 (2006).

[74] E. W. Streed, B. G. Norton, A. Jechow, T. J. Weinhold, and D. Kielpinski, Physical review letters 106, 010502 (2011).

[75] J. T. Merrill, C. Volin, D. Landgren, J. M. Amini, K. Wright, S. C. Doret, C. Pai, H. Hayden, T. Killian, D. Faircloth, et al., New Journal of Physics 13, 103005 (2011).

[76] M. Trupke, J. Goldwin, B. Darquié, G. Dutier, S. Eriksson, J. Ashmore, and E. Hinds, Physical review letters 99, 063601 (2007).

[77] E. Vetsch, D. Reitz, G. Sagué, R. Schmidt, S. Dawkins, and A. Rauschenbeutel, Physical review letters 104, 203603 (2010).

[78] G. Brambilla, F. Xu, P. Horak, Y. Jung, F. Koizumi, N. P. Sessions, E. Koukharenko, X. Feng, G. S. Murugan, J. S. Wilkinson, et al., Advances in Optics and Photonics 1, 107 (2009).

[79] K. Sakmann and M. Kasevich, Opt. Lett. 39, 5317 (2014).

[80] S. A. Knappe, H. G. Robinson, and L. Hollberg, Optics express 15, 6293 (2007).

[81] W. Yang, D. B. Conkey, B. Wu, D. Yin, A. R. Hawkins, and H. Schmidt, Nature Photonics 1, 331 (2007).

[82] S. Spillane, G. Pati, K. Salit, M. Hall, P. Kumar, R. Beausoleil, and M. Shahriar, Physical review letters 100, 233602 (2008).
[83] A. Sargsyan, D. Sarkisyan, A. Papoyan, Y. PashayanLeroy, P. Moroshkin, A. Weis, A. Khanbekyan, E. Mariotti, and L. Moi, Laser Physics 18, 749 (2008).

[84] W. D. Phillips and H. Metcalf, Physical Review Letters 48, 596 (1982).

[85] W. D. Phillips, J. V. Prodan, and H. J. Metcalf, JOSA B 2, 1751 (1985).

[86] T. Liebisch, E. Blanshan, E. Donley, and J. Kitching, Physical Review A 85, 013407 (2012).

[87] T. Arpornthip, C. Sackett, and K. Hughes, Physical Review A 85, 033420 (2012).

[88] P. Berthoud, A. Joyet, G. Dudle, N. Sagna, and P. Thomann, EPL (Europhysics Letters) 41, 141 (1998).

[89] D. A. Steck, "Rubidium 87 D line data," (2001).

[90] L.-A. Liew, S. Knappe, J. Moreland, H. Robinson, L. Hollberg, and J. Kitching, Applied Physics Letters 84, 2694 (2004).

[91] F. Vecchio, V. Venkatraman, H. Shea, T. Maeder, and P. Ryser, Procedia Engineering 5, 367 (2010).

[92] S. Radhakrishnan and A. Lal, in Solid-State Sensors, Actuators and Microsystems, 2005. Digest of Technical Papers. TRANSDUCERS'05. The 13th International Conference on, Vol. 1 (IEEE, 2005) pp. 23-26.

[93] L.-A. Liew, J. Moreland, and V. Gerginov, Applied physics letters 90, 114106 (2007).

[94] F. Gong, Y.-Y. Jau, K. Jensen, and W. Happer, Review of scientific instruments 77, 076101 (2006).

[95] S. Bartalini, I. Herrera, L. Consolino, L. Pappalardo, N. Marino, G. D'Arrigo, and F. Cataliotti, The European Physical Journal D-Atomic, Molecular, Optical and Plasma Physics 36, 101 (2005).

[96] Www.alvatec.com.

[97] P. Griffin, K. Weatherill, and C. Adams, Review of scientific instruments 76, 093102 (2005).

[98] A. Douahi, L. Nieradko, J. Beugnot, J. Dziuban, H. Maillote, S. Guerandel, M. Moraja, C. Gorecki, and V. Giordano, Electronics Letters 43, 33 (2007).

[99] Integrating high power focused beams into a chip scale system would not be trivial and the beam must be moved once sections of the dispenser are depleted. An alternative heating method could include induction heating.

[100] M. Stephens, R. Rhodes, and C. Wieman, Journal of applied physics 76, 3479 (1994).

[101] In that study they found that Pyrex took an order of magnitude longer to cure than sapphire. The glass used in this study is likely to have properties in between these materials.

[102] The effect of hard optical coatings on the glass, such as $\mathrm{MgF}_{2}$ to reduce reflections or ITO for heating, may also reduce the effectiveness of glass as a pump.

[103] H. Bottner, in Thermoelectrics, 2002. Proceedings ICT'02. Twenty-First International Conference on (IEEE, 2002) pp. 511-518.

[104] D. R. Scherer, D. B. Fenner, and J. M. Hensley, Journal of Vacuum Science \& Technology A: Vacuum, Surfaces, and Films 30, 061602 (2012).

[105] P. della Porta, C. Emili, and J. Hellier, in IEEE Conference on tube techniques (IEEE, 1968).

[106] Scherer et a 1[104] state that NEGs do not pump rubidium at all, whereas della Porta et al [105] state that alkali atoms do not affect the pumping speed for other gases. We suspect there is some pumping due to the high reactivity of alkali atoms with metals and with most ad- 
sorbed gases.

[107] A. Pelton, Bulletin of Alloy Phase Diagrams 7, 139 (1986).

[108] M. B. Squires, High repetition rate Bose-Einstein condensate production in a compact, transportable vacuum system, Ph.D. thesis, University of Colorado (2008).

[109] N. Bhaskar and C. Kahla, Ultrasonics, Ferroelectrics and Frequency Control, IEEE Transactions on 37, 355 (1990).

[110] P. V. Medeiros, F. de Brito Mota, A. J. Mascarenhas, and C. M. de Castilho, Nanotechnology 21, 115701 (2010).

[111] M. Caragiu and S. Finberg, Journal of Physics: Condensed Matter 17, R995 (2005).

[112] L. Torralbo-Campo, G. D. Bruce, G. Smirne, and D. Cassettari, arXiv preprint arXiv:1312.6442 (2013).

[113] A. Hatakeyama, M. Wilde, and K. Fukutani, arXiv preprint cond-mat/0512451 (2005).

[114] K. Rębilas and M. J. Kasprowicz, Physical Review A 79, 042903 (2009)

[115] S. Villalba, H. Failache, and A. Lezama, Physical Review A 82, 56902 (2010).

[116] S. Atutov, R. Calabrese, V. Guidi, B. Mai, A. Rudavets, E. Scansani, L. Tomassetti, V. Biancalana, A. Burchianti, C. Marinelli, et al., Physical Review A 67, 053401 (2003).

[117] B. Anderson and M. Kasevich, Physical Review A 63, 023404 (2001).

[118] D. Xiong, F. Wang, X. Li, T.-F. Lam, and D. Wang, arXiv preprint arXiv:1303.0333 (2013).

[119] S. Du, M. B. Squires, Y. Imai, L. Czaia, R. Saravanan, V. Bright, J. Reichel, T. Hänsch, and D. Z. Anderson, Physical Review A 70, 053606 (2004).

[120] A. Burchianti, C. Marinelli, A. Bogi, J. Brewer, K. Rubahn, H.-G. Rubahn, F. Della Valle, E. Mariotti, V. Biancalana, S. Veronesi, et al., EPL (Europhysics Letters) 67, 983 (2004).

[121] S. Villalba, H. Failache, and A. Lezama, Physical Review A 81, 032901 (2010).

[122] A. Pelton, Journal of Phase Equilibria 7, 228 (1986).

[123] J. Sangster and A. Pelton, Journal of phase equilibria 12, 447 (1991).

[124] A. Pelton, Bulletin of Alloy Phase Diagrams 7, 136 (1986).

[125] J. Sangster and A. Pelton, Journal of phase equilibria 12, 451 (1991)

[126] A. Pelton, Journal of Phase Equilibria 7, 23 (1986).

[127] A. Petric and A. Pelton, Journal of phase equilibria 12, 29 (1991).

[128] A. Pelton and A. Petric, Journal of phase equilibria 14 368 (1993).

[129] A. Pelton, Bulletin of Alloy Phase Diagrams 7, 19 (1986).

[130] J. Sangster and A. Pelton, Journal of phase equilibria 12, 443 (1991).

[131] J. F. O'Hanlon, A user's guide to vacuum technology (John Wiley \& Sons, 2005).

[132] V. Lindroos, M. Tilli, A. Lehto, and T. Motooka, Handbook of silicon based MEMS materials and technologies (Elsevier, 2009).

[133] W. Choi, B. Ju, Y. Lee, S. Jeong, N. Lee, M. Sung, and M. Oh, Journal of The Electrochemical Society 146, 400 (1999).

[134] B. Erjavec, Vacuum 64, 15 (2001).
[135] P. Willems and K. Libbrecht, Physical Review A 51, 1403 (1995).

[136] A. Roth, Vacuum sealing techniques (Springer, 1994).

[137] The lowest outgassing rates for metals are tabulated by Redhead [277] with high temperature annealed stainless steel and OFHC copper displaying outgassing rates between $10^{-15}$ and $10^{-16}$ mbar $1 \mathrm{~s}^{-1} \mathrm{~cm}^{-2}$.

[138] W. Perkins, Journal of Vacuum Science and Technology 10, 543 (1973).

[139] S.-w. Zhang, Z.-j. Zhang, C.-h. XU, and L. Wang, Journal of Northeastern University (Natural Science) 8, 027 (2008).

[140] To be more precise, the authors cannot find absolute leak rate of these seals in the literature and commercial glass-metal, or glass-ceramic, seals only state leak rates 'below $10^{-10} \mathrm{mbar} \mathrm{s}^{-1}$. However, our discussions with commercial vacuum tube manufacturers indicate that the leak rate for metal-ceramic seals could be as low as $10^{-18} \mathrm{mbarl}^{-1} \mathrm{~s}^{-1}$ or lower.

[141] H.-C. Chuang and C.-S. Huang, Review of Scientific Instruments 85, 053107 (2014).

[142] K. E. Petersen, Proceedings of the IEEE 70, 420 (1982).

[143] Http://www.silcotek.com/silcodtechnologies/SilcoGuard-high-purity-coating/.

[144] C. Dedman, K. Baldwin, and M. Colla, Review of Scientific Instruments 72, 4055 (2001).

[145] A. Besharatian, K. Kumar, R. L. Peterson, L. P. Bernal, and K. Najafi, in Micro Electro Mechanical Systems (MEMS), 2012 IEEE 25th International Conference on (IEEE, 2012) pp. 1001-1004.

[146] H. Zhou, H. Li, V. Sharma, and M. Schmidt, in Micro Electro Mechanical Systems (MEMS), 2011 IEEE 24th International Conference on (IEEE, 2011) pp. 10951098.

[147] S. An, N. K. Gupta, and Y. B. Gianchandani, (2014).

[148] W. Yang, "Radially staged microscale turbomolecular pump," (2007), uS Patent App. 11/859,682.

[149] V. Jayanty, Miniaturized electron-impact-ionization pumps using double-gated isolated vertically aligned carbon nanotube arrays, Ph.D. thesis, Massachusetts Institute of Technology (2012).

[150] S. R. Green, R. Malhotra, and Y. B. Gianchandani, Microelectromechanical Systems, Journal of 22, 309 (2013).

[151] D. Jang et al., Carbon nanotube-based field ionization vacuum, Ph.D. thesis, Massachusetts Institute of Technology (2012).

[152] D. Laser and J. Santiago, Journal of micromechanics and microengineering 14, R35 (2004).

[153] A. Górecka-Drzazga, Vacuum 83, 1419 (2009).

[154] For a more detailed up to date sub-division of getter types see Chuntonov et al [169].

[155] C. Benvenuti, J. Cazeneuve, P. Chiggiato, F. Cicoira, A. Escudeiro Santana, V. Johanek, V. Ruzinov, and J. Fraxedas, Vacuum 53, 219 (1999).

[156] R. Sharma, N. Mithal, K. Bhushan, D. Srivastava, H. Prabhakara, S. Gadkari, J. Yakhmi, V. Sahni, et al., in Journal of Physics: Conference Series, Vol. 114 (IOP Publishing, 2008) p. 012050.

[157] P. Chiggiato and P. Costa Pinto, Thin Solid Films 515, 382 (2006).

[158] M. Bender, H. Kollmus, M. Bellachioma, and W. Assmann, Nuclear Instruments and Methods in Physics Research Section B: Beam Interactions with Materials and 
Atoms 268, 1986 (2010).

[159] K. Jousten, Handbook of vacuum technology (John Wiley \& Sons, 2008).

[160] C. Benvenuti, P. Chiggiato, F. Cicoira, and V. Ruzinov, Vacuum 50, 57 (1998).

[161] A. Prodromides, C. Scheuerlein, and M. Taborelli, Vacuum 60, 35 (2001).

[162] C. Benvenuti, A. Escudeiro Santana, and V. Ruzinov, Vacuum 60, 279 (2001).

[163] A. Prodromides and F. Lévy, Non-Evaporable Getter Thin Film Coatings for Vacuum Applications, Tech. Rep. (CERN, 2002).

[164] SAES getters and Nanogetters.

[165] S. Amorosi, M. Anderle, C. Benvenuti, S. Calatroni, J. Carver, P. Chiggiato, H. Neupert, and W. Vollenberg, Vacuum 60, 89 (2001).

[166] B. Window, Journal of Vacuum Science \& Technology A: Vacuum, Surfaces, and Films 11, 1522 (1993).

[167] V. Chidambaram, X. Ling, and C. Bangtao, Journal of electronic materials 42, 485 (2013).

[168] C. Liu, L. Shi, S. Xu, Z. Zhou, S. Luo, and X. Long, Vacuum 75, 71 (2004).

[169] K. A Chuntonov and S. P Yatsenko, Recent Patents on Materials Science 6, 29 (2013).

[170] K. Chuntonov and J. Setina, Journal of Alloys and Compounds 455, 489 (2008).

[171] K. Chuntonov, A. Ivanov, and D. Permikin, Journal of Alloys and Compounds 456, 187 (2008).

[172] K. Chuntonov, J. Setina, A. Ivanov, and D. Permikin, Journal of Alloys and Compounds 460, 357 (2008).

[173] K. Chuntonov, A. Ivanov, and D. Permikin, Journal of Alloys and Compounds 471, 211 (2009).

[174] J. Sangster and A. Pelton, Journal of phase equilibria 15, 87 (1994).

[175] One could consider using lithium as the trapped species instead.

[176] J. F. Shackelford, P. L. Studt, and R. M. Fulrath, Journal of Applied Physics 43, 1619 (1972).

[177] A common value quoted in the literature is the 'characteristic time' $t_{c}=\frac{d^{2}}{6 D}$, this is time it takes to produce a constant flow, not for the gas to initially break through and so overestimates the time it take for the permeating gas to effect the vacuum.

[178] W. Rogers, R. Buritz, and D. Alpert, Journal of Applied Physics 25, 868 (1954).

[179] V. Altemose, Journal of Applied Physics 32, 1309 (1961).

[180] J. Covino and J. M. Bennett, Laser-Gyro Materials Studies, Tech. Rep. (DTIC Document, 1986).

[181] K. Knowles and A. Van Helvoort, International materials reviews 51, 273 (2006).

[182] J. Shelby and R. J. Eagan, Journal of the American Ceramic Society 59, 420 (1976).

[183] G. Wallis and D. I. Pomerantz, Journal of Applied Physics 40, 3946 (1969).

[184] P. Baine, L. Quinn, B. Lee, S. Mitchell, B. Armstrong, and H. Gamble, Thin solid films 296, 141 (1997).

[185] L. Spangler and K. Wise, in Solid-State Sensor and Actuator Workshop, 1988. Technical Digest., IEEE (IEEE, 1988) pp. 140-143.

[186] P. M. Sarro, Sensors and Actuators A: Physical 82, 210 (2000).

[187] P. Jung, Journal of nuclear materials 191, 377 (1992).
[188] C. Tudryn, S. Schweizer, R. Hopkins, L. Hobbs, and A. J. Garratt-Reed, Journal of the Electrochemical Society 152, E131 (2005).

[189] A. Shevchenko, M. Heiliö, T. Lindvall, A. Jaakkola, I. Tittonen, M. Kaivola, and T. Pfau, Physical Review A 73, 051401 (2006).

[190] P. Trocellier, S. Agarwal, and S. Miro, Journal of Nuclear Materials 445, 128 (2014).

[191] J. S. Bunch, S. S. Verbridge, J. S. Alden, A. M. van der Zande, J. M. Parpia, H. G. Craighead, and P. L. McEuen, Nano letters 8, 2458 (2008).

[192] R. Nair, H. Wu, P. Jayaram, I. Grigorieva, and A. Geim, Science 335, 442 (2012).

[193] A. Van Wieringen and N. Warmoltz, Physica 22, 849 (1956).

[194] M. Alatalo, M. Puska, and R. Nieminen, Physical Review B 46, 12806 (1992).

[195] P. Souers, I. Moen, R. Lindahl, and R. Tsugawa, Journal of the American Ceramic Society 61, 42 (1978).

[196] S. Steward, Review of hydrogen isotope permeability through materials, Tech. Rep. (Lawrence Livermore National Lab., CA (USA), 1983).

[197] N. Kurita, N. Fukatsu, H. Otsuka, and T. Ohashi, Solid state ionics 146, 101 (2002).

[198] H. Laska, R. Doremus, and P. Jorgensen, The Journal of Chemical Physics 50, 135 (1969).

[199] F. J. Norton, Journal of Applied Physics 28, 34 (1957).

[200] P. Marin, M. Dialinas, G. Lissillour, A. Marraud, and A. Reboux, Vacuum 49, 309 (1998).

[201] P. Jung, Nuclear Instruments and Methods in Physics Research Section B: Beam Interactions with Materials and Atoms 91, 362 (1994).

[202] C. Griffioen, J. Evans, P. D. Jong, and A. V. Veen, Nuclear Instruments and Methods in Physics Research Section B: Beam Interactions with Materials and Atoms 27, 417 (1987).

[203] C.-A. Chen, Y. Sun, Z. Huang, C. Liu, and S. Wu, Physica Scripta 2003, 97 (2003).

[204] M. Mantina, A. C. Chamberlin, R. Valero, C. J. Cramer, and D. G. Truhlar, The Journal of Physical Chemistry A 113, 5806 (2009).

[205] S. Batsanov, Inorganic materials 37, 871 (2001).

[206] M. Binns, S. McQuaid, R. Newman, and E. Lightowlers, Semiconductor science and technology 8, 1908 (1993).

[207] D. Sparks, Journal of Micromechanics and Microengineering 23, 015016 (2013).

[208] D. Sparks, J. Mitchell, and S. Lee, Journal of Sensor Technology 3, 101 (2013).

[209] G. F. Cerofolini, F. Corni, S. Frabboni, C. Nobili, G. Ottaviani, and R. Tonini, Materials Science and Engineering: R: Reports 27, 1 (2000).

[210] Ultra low helium leak detectors using the accumulation method and NEG pumps have measured leak rates [304] as low as $10^{-15} \mathrm{mbarls}^{-1}$.

[211] S. H. Christiansen, R. Singh, and U. Gosele, Proceedings of the IEEE 94, 2060 (2006).

[212] The relative conductance, or leak rate, of noninteracting gases, is proportional to the square root of their masses. Hence helium will leak 2.6 times faster than nitrogen. For the following calculation we has assumed the average molecular mass of is 29 .

[213] G. Attard and C. Barnes, Surfaces (Oxford University Press Oxford, UK, 1998). 
[214] R. Straessle, M. Pellaton, C. Affolderbach, Y. Pétremand, D. Briand, G. Mileti, and N. de Rooij, Journal of Applied Physics 113, 064501 (2013).

[215] D. Sparks, S. Massoud-Ansari, and N. Najafi, in Micromachining and Microfabrication (International Society for Optics and Photonics, 2004) pp. 70-78.

[216] D. Sparks, J. Trevino, S. Massoud-Ansari, and N. Najafi, Journal of Micromechanics and Microengineering 16, 2488 (2006).

[217] R. Wolffenbuttel and K. Wise, Sensors and Actuators A: Physical 43, 223 (1994).

[218] S. Lani, A. Bosseboeuf, B. Belier, C. Clerc, C. Gousset, and J. Aubert, Microsystem technologies 12, 1021 (2006).

[219] C. C. Lee, C. Y. Wang, and G. Matijasevic, Components, Hybrids, and Manufacturing Technology, IEEE Transactions on 16, 311 (1993).

[220] This is not the case for Au-Sn eutectic as the formation of the alloy increases the eutectic point.

[221] S. Mack, H. Baumann, U. Gösele, H. Werner, and R. Schlögl, Journal of the Electrochemical Society 144, 1106 (1997).

[222] S. Mack, H. Baumann, and U. Gosele, in Micro Electro Mechanical Systems, 1997. MEMS'97, Proceedings, IEEE., Tenth Annual International Workshop on (IEEE, 1997) pp. 488-493.

[223] U. Gosele, H. Stenzel, T. Martini, J. Steinkirchner, D. Conrad, and K. Scheerschmidt, Applied physics letters 67, 3614 (1995).

[224] T.-K. Chuang, A. Usenko, and J. Cites, ECS Transactions 33, 501 (2010).

[225] S. Millar and M. Desmulliez, Sensor Review 29, 339 (2009).

[226] W. Reinert, D. Kahler, and G. Longoni, in Electronic Packaging Technology Conference, 2005. EPTC 2005. Proceedings of 7 th, Vol. 1 (IEEE, 2005) pp. 6-pp.

[227] Z. Gan, D. Huang, X. Wang, D. Lin, and S. Liu, Sensors and Actuators A: Physical 149, 159 (2009).

[228] S.-W. Choi, W.-B. Choi, Y.-H. Lee, B.-K. Ju, M.-Y. Sung, and B.-H. Kim, Journal of The Electrochemical Society 149, G8 (2002).

[229] O. Zucker, W. Langheinrich, M. Kulozik, and H. Goebel, Sensors and Actuators A: Physical 36, 227 (1993).

[230] W. Kern, Journal of the Electrochemical Society 137, 1887 (1990).

[231] W. Kern et al., Handbook of semiconductor wafer cleaning technology, Vol. 623 (Noyes Park Ridge, NJ, 1993).

[232] J. R. Vig, Journal of Vacuum Science \& Technology A: Vacuum, Surfaces, and Films 3, 1027 (1985).

[233] S. Franssila, Introduction to microfabrication (Wiley. com, 2010).

[234] M. Taborelli, Cleaning and surface properties, Tech. Rep. (CERN, 2006).

[235] We assume a hydrogen capacity of $10^{-7}$ mols $\left(x_{H}=\right.$ $0.01)$ which results in $Q_{O G} \sim 10^{-12} \mathrm{mbarls}^{-1} \mathrm{~cm}^{-2}$.

[236] R. Elsey, Vacuum 25, 299 (1975).

[237] R. Elsey, Vacuum 25, 347 (1975).

[238] Typically $k=1$ for high surface coverage, but increases to 2 when desorption is recombination limited at low coverage.

[239] G. S. Lewin, Fundamentals of Vacuum Science and Technology (McGraw-Hill, USA, 1965).

[240] W. Perkins and D. Begeal, The Journal of Chemical
Physics 54, 1683 (1971).

[241] V. Nemanič, P. J. McGuiness, N. Daneu, B. Zajec, Z. Siketić, and W. Waldhauser, Journal of alloys and compounds 539, 184 (2012).

[242] N. Nickel, W. Jackson, I. Wu, C. Tsai, and A. Chiang, Physical Review B 52, 7791 (1995).

[243] S. Koveshnikov, S. Nosenko, and E. Yakimov, physica status solidi (a) 120, 391 (1990).

[244] Y. Kamiura, M. Yoneta, and F. Hashimoto, Applied physics letters 59, 3165 (1991).

[245] C. Seager and R. Anderson, Applied physics letters 53, 1181 (1988).

[246] K. Schmalz and K. Tittelbach-Helmrich, physica status solidi (a) 113, K9 (1989).

[247] M. Vergnat, S. Houssaini, C. Dufour, A. Bruson, G. Marchal, P. Mangin, R. Erwin, J. Rhyne, and C. Vettier, EPL (Europhysics Letters) 14, 457 (1991).

[248] X.-M. Tang, J. Weber, Y. Baer, and F. Finger, Physical Review B 42, 7277 (1990).

[249] N. Johnson, C. Nebel, P. Santos, W. Jackson, R. Street, K. Stevens, and J. Walker, Applied physics letters 59, 1443 (1991).

[250] C. Seager, R. Anderson, and D. Brice, Journal of applied physics 68, 3268 (1990).

[251] R. Newman, J. Tucker, A. Brown, and S. McQuaid, Journal of applied physics 70, 3061 (1991).

[252] A. Majumdar, S. Balasubramanian, V. Venkataraman, and N. Balasubramanian, Journal of applied physics $\mathbf{8 2}$, 192 (1997).

[253] B. Sopori, X. Deng, J. Benner, A. Rohatgi, P. Sana, S. Estreicher, Y. Park, and M. Roberson, Solar Energy Materials and Solar Cells 41, 159 (1996).

[254] C. Herrero, M. Stutzmann, A. Breitschwerdt, and P. Santos, Physical Review B 41, 1054 (1990).

[255] N. Johnson and C. Herring, Physical Review B 46, 15554 (1992).

[256] R. Rizk, P. De Mierry, D. Ballutaud, M. Aucouturier, and D. Mathiot, Physical review B 44, 6141 (1991).

[257] S. Fabian, S. Kalbitzer, C. Klatt, M. Behar, and C. Langpape, Physical Review B 58, 16144 (1998).

[258] Y. Huang, Y. Ma, R. Job, and A. Ulyashin, Journal of applied physics 96, 7080 (2004).

[259] W. Hansen, S. Pearton, and E. Haller, Applied Physics Letters 44, 606 (1984).

[260] M. Capizzi and A. Mittiga, Applied physics letters 50, 918 (1987).

[261] A. Mogro-Campero, R. Love, and R. Schubert, Journal of The Electrochemical Society 132, 2006 (1985).

[262] S. J. Pearton, J. W. Corbett, and T. Shi, Applied Physics A 43, 153 (1987).

[263] J. Chevallier and M. Aucouturier, Annual Review of Materials Science 18, 219 (1988).

[264] E. E. Haller, Semiconductor Science and Technology 6, 73 (1991).

[265] S. Myers, H. Birnbaum, J. Corbett, G. DeLeo, S. Estreicher, E. Haller, P. Jena, N. Johnson, R. Kirchheim, and S. Pearton, Reviews of Modern Physics 64, 559 (1992).

[266] C. G. Van de Walle and J. Neugebauer, Annu. Rev. Mater. Res. 36, 179 (2006).

[267] T. Miyake, T. Ogitsu, and S. Tsuneyuki, Physical review letters 81, 1873 (1998).

[268] B. Sopori, Y. Zhang, and N. Ravindra, Journal of electronic materials 30, 1616 (2001).

[269] T. Ichimiya and A. Furuichi, The International Journal 
of Applied Radiation and Isotopes 19, 573 (1968).

[270] S. McQuaid, R. Newman, J. Tucker, E. Lightowlers, R. A. Kubiak, and M. Goulding, Applied physics letters 58, 2933 (1991).

[271] Even higher surface concentrations can be produced with high energy ion bombardment, but we regard this as a non-standard process.

[272] M. Dürr and U. Höfer, Surface science reports 61, 465 (2006).

[273] P. Gupta, V. Colvin, and S. George, Physical Review B 37, 8234 (1988).

[274] T. Hickmott, Journal of Applied Physics 31, 128 (1960).

[275] B. J. Todd, Journal of Applied Physics 26, 1238 (1955).

[276] J. Hobson, Journal of Vacuum Science and Technology 1, 1 (1964).

[277] P. Redhead, Vacuum 53, 137 (1999).

[278] Hobson [276] measured a 'leak rate' (which we assume to be outgassing) of $1.8 \times 10^{-14}$ Torr $1 \mathrm{~s}^{-1}$ (equiv. nitrogen) but gave no value for the glass surface area other than it being similar to Redhead et al's apparatus [282] who used a $500 \mathrm{ml}$ bulb. Assuming the surface area of $300 \mathrm{~cm}^{2}$ we calculate an outgassing rate of $8 \times 10^{-17} \mathrm{mbarl} \mathrm{s}^{-1} \mathrm{~cm}^{-2}$.

[279] This appears counter-intuitive, but the greater pressure results in more collisions and thermalization with the surrounding gas atoms which reduces the velocity and, therefore, the probability of being buried in the growing film.

[280] G.-Y. Hsiung, C. Cheng, C. Yang, C. Chan, and J. Chen, in AIP Conference Proceedings (2009).

[281] W. Palosz, Journal of crystal growth 267, 475 (2004).

[282] P. Redhead, E. Kornelsen, and J. Hobson, Canadian Journal of Physics 40, 1814 (1962).

[283] W. Lange, Journal of Vacuum Science and Technology 2, 74 (1965).

[284] R. Baptist and F. Levy, Vacuum 43, 213 (1992).

[285] J. Bennett, S. Hughes, R. Elsey, and T. Parry, Vacuum 73, 149 (2004).

[286] D. Hu, W. Ho, X. Chen, S. Wang, and W. A. Goddard III, Physical review letters 78, 1178 (1997).

[287] J. Setina, S. Avdiaj, and B. Erjavec, Vacuum 92, 20 (2013).

[288] J. Kamiya, V. Baglin, G. Bregliozzi, and J. M. Jimenez,
Vacuum 85, 1178 (2011).

[289] M. Hasegawa, R. K. Chutani, R. Boudot, L. Mauri, C. Gorecki, X. Liu, and N. Passilly, Journal of Micromechanics and Microengineering 23, 055022 (2013).

[290] H. Henmi, S. Shoji, Y. Shoji, K. Yoshimi, and M. Esashi, Sensors and Actuators A: Physical 43, 243 (1994).

[291] T. Rogers, Journal of Micromechanics and Microengineering 2, 164 (1992).

[292] S. Shoji, H. Kikuchi, and H. Torigoe, Sensors and Actuators A: Physical 64, 95 (1998).

[293] J. Wei, H. Xie, M. Nai, C. Wong, and L. Lee, Journal of Micromechanics and Microengineering 13, 217 (2003).

[294] A. Gerlach, D. Maas, D. Seidel, H. Bartuch, S. Schundau, and K. Kaschlik, Microsystem technologies 5, 144 (1999).

[295] U. Mescheder, M. Alavi, K. Hiltmann, C. Lietzau, C. Nachtigall, and H. Sandmaier, Sensors and Actuators A: Physical 97, 422 (2002).

[296] H. Takagi, R. Maeda, T. R. Chung, and T. Suga, Sensors and Actuators A: Physical 70, 164 (1998).

[297] V. Dragoi, G. Mittendorfer, C. Thanner, and P. Lindner, Microsystem Technologies 14, 509 (2008).

[298] V. Nemanič and J. Šetina, Vacuum 49, 233 (1998).

[299] H. Wensink, J. Berenschot, H. V. Jansen, and M. C. Elwenspoek, in Micro Electro Mechanical Systems, 2000. MEMS 2000. The Thirteenth Annual International Conference on (IEEE, 2000) pp. 769-774.

[300] B. Jian and W. A. van Wijngaarden, JOSA B 30, 238 (2013).

[301] Y. Ishikawa and T. Yoshimura, Journal of Vacuum Science \& Technology A: Vacuum, Surfaces, and Films 13, 1847 (1995).

[302] N. Robinson, The Physical Principles of Ultra-high Vacuum: Systems and Equipment, High vacuum series (Chapman and Hall, 1968).

[303] H. Ohadi, M. Himsworth, A. Xuereb, and T. Freegarde, Optics express 17, 23003 (2009).

[304] L. E. Bergquist and Y. T. Sasaki, Journal of Vacuum Science \& Technology A: Vacuum, Surfaces, and Films 10, 2650 (1992). 